TRANSACTIONS OF THE

AMERICAN MATHEMATICAL SOCIETY

Volume 360, Number 6, June 2008, Pages 2825-2847

S 0002-9947(08)04208-6

Article electronically published on January 4, 2008

\title{
INTEGRAL HOMOLOGY 3-SPHERES AND THE JOHNSON FILTRATION
}

\author{
WOLFGANG PITSCH
}

\begin{abstract}
The mapping class group of an oriented surface $\Sigma_{g, 1}$ of genus $g$ with one boundary component has a natural decreasing filtration $\mathcal{M}_{g, 1} \supset$ $\mathcal{M}_{g, 1}(1) \supset \mathcal{M}_{g, 1}(2) \supset \mathcal{M}_{g, 1}(3) \supset \cdots$, where $\mathcal{M}_{g, 1}(k)$ is the kernel of the action of $\mathcal{M}_{g, 1}$ on the $k^{t h}$ nilpotent quotient of $\pi_{1}\left(\Sigma_{g, 1}\right)$. Using a tree Lie algebra approximating the graded Lie algebra $\bigoplus_{k} \mathcal{M}_{g, 1}(k) / \mathcal{M}_{g, 1}(k+1)$ we prove that any integral homology sphere of dimension 3 has for some $g$ a Heegaard decomposition of the form $M=\mathcal{H}_{g} \coprod_{\iota_{g} \phi}-\mathcal{H}_{g}$, where $\phi \in \mathcal{M}_{g, 1}(3)$ and $\iota_{g}$ is such that $\mathcal{H}_{g} \coprod_{\iota_{g}}-\mathcal{H}_{g}=S^{3}$. This proves a conjecture due to $\mathrm{S}$. Morita and shows that the "core" of the Casson invariant is indeed the Casson invariant.
\end{abstract}

\section{INTRODUCTION}

In the early 80's, A. Casson succeeded to lift the classical Rohlin invariant of integral homology 3 -spheres, which is $\mathbf{Z} / 2 \mathbf{Z}$-valued, to a $\mathbf{Z}$-valued invariant. Later S. Morita [11] used the theory of Heegaard splittings combined with techniques from group cohomology to give a new construction of this invariant related to algebraic properties of the mapping class group of an oriented surface.

More precisely, let $\Sigma_{g}$ be a closed oriented surface of genus $g$. For technical reasons, fix a small disc $D^{2}$ on $\Sigma_{g}$ and consider the mapping class group $\mathcal{M}_{g, 1}$, i.e. the group of orientation preserving diffeomorphisms of $\Sigma_{g}$ which are the identity on $D^{2}$ modulo isotopies which are also the identity on $D^{2}$. Let $h: \Sigma_{g} \rightarrow \mathbf{S}^{3}$ be a fixed Heegaard embedding of $\mathbf{S}^{3}$, and for $\phi \in \mathcal{M}_{g, 1}$ let $\mathbf{S}_{\phi}^{3}$ denote the manifold obtained by cutting $\mathbf{S}^{3}$ along $h\left(\Sigma_{g}\right)$ and gluing back the two pieces by a map representing $\phi$. It is a classical fact ([7], chap. 8) that the diffeomorphism class of $\mathbf{S}_{\phi}^{3}$ is well-defined, and that any diffeomorphism class of closed oriented 3-manifolds can be obtained in this way.

The mapping class group has a natural filtration by normal subgroups, called the Johnson filtration: $\cdots \mathcal{M}_{g, 1}(k+1) \subset \mathcal{M}_{g, 1}(k) \subset \cdots \mathcal{M}_{g, 1}$, and it is a natural question to try to relate this filtration to the theory of Heegaard splittings. For instance, if we restrict the map $\phi \mapsto \mathbf{S}_{\phi}^{3}$ to the group $\mathcal{M}_{g, 1}(1)$, also called the Torelli group, what we get are exactly the integral homology 3 -spheres $\mathcal{S}(3)$. In [11] S. Morita proved that the elements of $\mathcal{M}_{g, 1}(2)$ suffice to obtain the whole set

Received by the editors November 7, 2005.

2000 Mathematics Subject Classification. Primary 57M99; Secondary 20F38, $20 \mathrm{~F} 12$.

The author was supported by MEC grant MTM2004-06686 and by the program Ramón y Cajal, MEC, Spain. 
$\mathcal{S}(3)$. Then he proved that the composite of the map $\mathcal{M}_{g, 1}(2) \rightarrow \mathcal{S}(3)$ with the Casson invariant $\mathcal{S}(3) \rightarrow \mathbf{Z}$ is a homomorphism of groups, and moreover that it is the sum of two morphisms $d$ and $q$. The first morphism $d$, which is related to cohomological properties of the mapping class group [16], was called by S. Morita "the core of the Casson invariant", and the second term $q$ appeared as a correcting term and was shown by S. Morita to vanish on the subgroup $\mathcal{M}_{g, 1}(3)$. Therefore he asked if this latter group suffices to construct all integral homology spheres. The purpose of this paper is to give an affirmative answer to this question:

Main Theorem. Any integral homology sphere is diffeomorphic to $S_{\phi}^{3}$ for some $\phi \in \mathcal{M}_{g, 1}(3)$ with $g \geq 9$.

Our starting point is a classical theorem of Singer. Roughly speaking it asserts that the sentence "the manifolds $S_{\phi}^{3}$ and $S_{\psi}^{3}$ are diffeomorphic" can be algebraically translated as "the mapping classes $\phi$ and $\psi$ lie in the same double coset in $\mathcal{A}_{g+h, 1} \backslash \mathcal{M}_{g+h, 1} / \mathcal{B}_{g+h, 1}$ for all sufficiently large $h$ ", where the subgroups $\mathcal{A}_{g, 1}$ and $\mathcal{B}_{g, 1}$ are known. Unfortunately, this algebraic statement is difficult to handle in practice. Therefore, we first construct an injective homomorphism $\tilde{\tau}_{2}$ from $\mathcal{M}_{g, 1}(2) / \mathcal{M}_{g, 1}(3)$ to a commutative group $\mathcal{A}_{2}\left(N_{1}\right)$. By commutativity, to check that an element in $\mathcal{M}_{g, 1}(2)$ is equivalent to some element in $\mathcal{M}_{g, 1}(3)$ it suffices then to prove that $\tilde{\tau}_{2}(\phi)$ belongs to the subgroup $\operatorname{Can}\left(\mathcal{A}_{2}\left(N_{1}\right)\right) \subset \mathcal{A}_{2}\left(N_{1}\right)$ generated by the images of $\left(\mathcal{M}_{g, 1}(2) \cap \mathcal{A}_{g, 1}\right) / \mathcal{M}_{g, 1}(3)$ and $\left(\mathcal{M}_{g, 1}(2) \cap \mathcal{B}_{g, 1}\right) / \mathcal{M}_{g, 1}(3)$.

This paper is organised as follows. In section 2 we have compiled some standard facts about mapping class groups, the Johnson filtration and Heegaard decompositions. In section 3 we introduce Lie algebra structures on the graded group $\bigoplus_{k} \mathcal{M}_{g, 1}(k) / \mathcal{M}_{g, 1}(k+1)$ and on the natural target of the Johnson's homomorphisms $\bigoplus_{k} D_{k}\left(N_{1}\right)$. We also introduce a graded injective homomorphism $\bigoplus_{k} \tilde{\tau}_{k}: \bigoplus_{k} D_{k}\left(N_{1}\right) \rightarrow \bigoplus_{k} \mathcal{A}_{k}\left(N_{1}\right)$ into a Lie algebra of labelled trees. In section 4 we give some properties of the group $\operatorname{Can}\left(\mathcal{A}_{2}\left(N_{1}\right)\right)$, in particular we study its invariance under some natural actions. The final section, section 5 , is devoted to the proof of the Main Theorem.

\section{Preliminaries}

2.1. Johnson's homomorphisms. Let $\mathcal{H}_{1,2}$ be an oriented solid torus with two small discs $D_{1}^{2}$ and $D_{2}^{2}$ on its boundary intersecting in one point $x_{0}$, and fix two based loops on the boundary: a parallel $\alpha$ and a meridian $\beta$, as in Figure 1.

Gluing $g$ copies of $\mathcal{H}_{1,2}$ by identifying $\left(D_{2}^{2}, x_{0}\right)$ on copy $i$ to $\left(D_{1}^{2}, x_{0}\right)$ on copy $i+1$ we get an oriented genus $g$ handlebody $\mathcal{H}_{g}$ with a small disc $D^{2}$ on its surface (the $D_{2}^{2}$ of the $g^{\text {th }}$ term of the sum). Let $\Sigma_{g}$ denote the boundary of $\mathcal{H}_{g}$, and $\Sigma_{g, 1}=\Sigma_{g} \backslash \stackrel{\circ}{D^{2}}$; these two oriented surfaces are naturally pointed by $x_{0}$. By construction the $2 g$ curves $\alpha_{i}, \beta_{i}$ (see Figure 2), which are the images of the different curves $\alpha$ and $\beta$, are free generators of the free group $\pi_{1}\left(\Sigma_{g, 1}, x_{0}\right)$.

Consider the central series of the fundamental group $\pi_{1}\left(\Sigma_{g, 1}, x_{0}\right)$, inductively defined by $\Gamma_{0}=\pi_{1}\left(\Sigma_{g, 1}, x_{0}\right)$, and $\forall k \geq 1 \Gamma_{k+1}=\left[\Gamma_{k}, \Gamma_{0}\right]$. Denote by $N_{k}$ the nilpotent quotient $\Gamma_{0} / \Gamma_{k}$. For instance $N_{1}$ is isomorphic to the abelianization of $\pi_{1}\left(\Sigma_{g, 1}, x_{0}\right)$.

Let $\mathcal{M}_{g, 1}$ denote the group of isotopy classes of diffeomorphisms which fix $D^{2}$ point-wise modulo isotopies which also fix $D^{2}$ point-wise, also called the mapping class group. Note that as they fix $D^{2}$ these mapping classes preserve the orientation. 


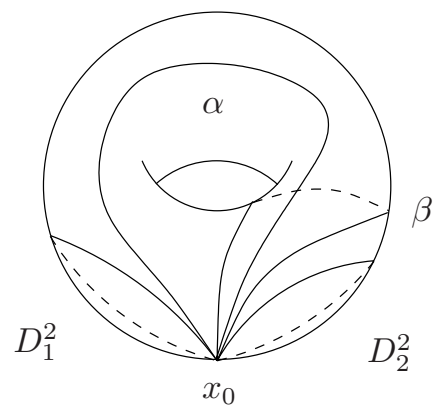

Figure 1. The torus $\mathcal{H}_{1,2}$

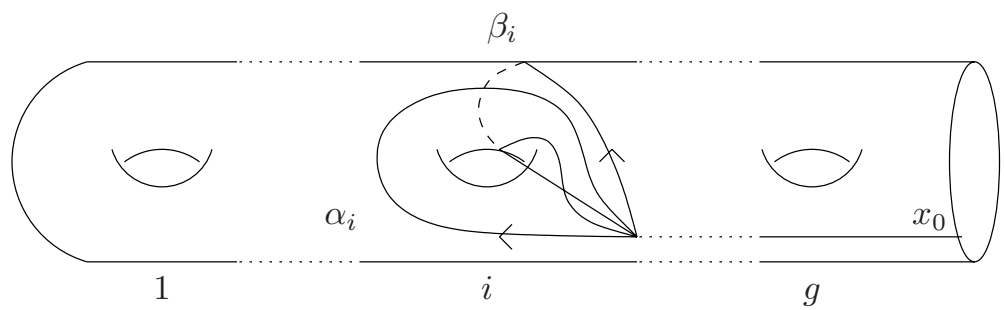

Figure 2. Model for $\Sigma_{g, 1}$

The natural action of $\mathcal{M}_{g, 1}$ on $\pi_{1}\left(\Sigma_{g, 1}, x_{0}\right)$ was described by Nielsen ([13], [14], [15]):

Theorem 2.1. The canonical morphism

$$
\mathcal{M}_{g, 1} \longrightarrow \operatorname{Aut}\left(\pi_{1}\left(\Sigma_{g, 1}, x_{0}\right)\right)
$$

is injective. Its image consists of those automorphisms which fix $\prod_{i=1}^{g}\left[\alpha_{i}, \beta_{i}\right]$.

As the commutator subgroups $\Gamma_{k}$ are preserved by any automorphism of $\pi_{1}\left(\Sigma_{g, 1}, x_{0}\right)$, the above action induces a family of compatible morphisms:

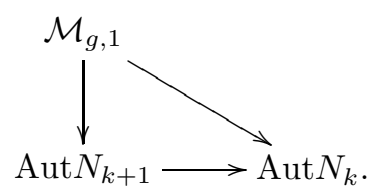

There is a canonical surjection $N_{k+1} \longrightarrow N_{k}$ whose kernel $\mathcal{L}_{k+1}$ is known to be the centre of $N_{k+1}$ ([10] p. 343). As the centre of a group is preserved by any automorphism one can prove (cf. [17]) that the central extension

$$
0 \longrightarrow \mathcal{L}_{k+1} \longrightarrow N_{k+1} \longrightarrow N_{k} \longrightarrow 1
$$

induces an exact sequence

$$
0 \longrightarrow \operatorname{Hom}\left(N_{1}, \mathcal{L}_{k+1}\right) \longrightarrow \operatorname{Aut}\left(N_{k+1}\right) \longrightarrow \operatorname{Aut}\left(N_{k}\right) \longrightarrow 1 .
$$


Let $\mathcal{M}_{g, 1}(k)$ denote the kernel of the canonical homomorphism $\mathcal{M}_{g, 1} \longrightarrow \operatorname{Aut}\left(N_{k}\right)$. We have an induced morphism, called the Johnson homomorphism $\tau_{k}: \mathcal{M}_{g, 1}(k) \longrightarrow$ $\operatorname{Hom}\left(N_{1}, \mathcal{L}_{k+1}\right)$. Explicitly, $\tau_{k}(f)$ is given as follows:

If $c \in N_{1}$ lifts to $\gamma \in \pi_{1}\left(\Sigma_{g, 1}, x_{0}\right)$, then

$$
\tau_{k}(f)(c)=f(\gamma) \gamma^{-1} \bmod \Gamma_{k+1} .
$$

The mapping class group acts on the kernels $\mathcal{M}_{g, 1}(k)$ by conjugation and on $\operatorname{Hom}\left(N_{1}, \mathcal{L}_{k+1}\right)$ through the conjugation action by $\operatorname{Aut}\left(N_{k+1}\right)$. These actions are obviously compatible, so that:

Proposition 2.1. The collection of Johnson homomorphisms reassemble into a monomorphism of graded groups and $\mathcal{M}_{g, 1}$-modules

$$
\bigoplus_{k \geq 1} \tau_{k}: \bigoplus_{k \geq 1} \mathcal{M}_{g, 1}(k) / \mathcal{M}_{g, 1}(k+1) \longrightarrow \bigoplus_{k \geq 1} \operatorname{Hom}\left(N_{1}, \mathcal{L}_{k+1}\right) .
$$

Recall that the algebraic intersection of closed paths on $\pi_{1}\left(\Sigma_{g, 1}, x_{0}\right)$ induces a symplectic form $\omega: \Lambda^{2} N_{1} \rightarrow \mathbf{Z}$, where we identify $N_{1}$ with $\mathrm{H}_{1}\left(\Sigma_{g, 1}\right)$ via the Hurewicz homomorphism. The image of the free basis $\alpha_{1}, \beta_{1} \ldots \alpha_{g}, \beta_{g}$ in $N_{1}$ is a symplectic basis $a_{1}, b_{1} \ldots a_{g}, b_{g}$ for $\omega$, and the image of the canonical morphism $\mathcal{M}_{g, 1} \rightarrow$ Aut $N_{1}$ is known to be the associated symplectic group $\operatorname{Sp} \omega$ (see [10] p. 178; the proof for the one boundary component case is the same). In the sequel we will denote the two Lagrangians generated by $\left\{a_{i} \mid 1 \leq i \leq g\right\}$ and $\left\{b_{i} \mid 1 \leq i \leq g\right\}$, respectively by $L_{A}$ and $L_{B}$. By definition we have a decomposition by $\omega$-dual Lagrangians $N_{1}=L_{A} \oplus L_{B}$.

2.2. Heegaard splittings. Let $\mathcal{V}(3)$ denote the set of all diffeomorphism classes of closed oriented 3 -manifolds and $\mathcal{S}(3)$ the subset of all integral homology spheres. By virtue of the classical theorem of Heegaard splittings, one can describe these two sets as the direct limit of a system of double cosets of mapping class groups.

Our inductive construction $\mathcal{H}_{g+1}=\mathcal{H}_{g} \sharp \mathcal{H}_{1,2}$ makes $\Sigma_{g, 1}$ a canonical subsurface of $\Sigma_{g+1,1}$, thus extending an orientation preserving diffeomorphism of $\Sigma_{g, 1}$ by the identity over its complement we get a well defined morphism $\mathcal{M}_{g, 1} \longrightarrow \mathcal{M}_{g+1,1}$ compatible with the action on the fundamental group. The Nielsen theorem implies that this morphism is injective.

Consider the following morphism:

$$
\begin{aligned}
\tilde{\iota}_{g}: \pi_{1}\left(\Sigma_{g, 1}, x_{0}\right) & \longrightarrow \pi_{1}\left(\Sigma_{g, 1}, x_{0}\right), \\
\alpha_{i} & \longmapsto \beta_{i}^{-1}, \\
\beta_{i} & \longmapsto \beta_{i} \alpha_{i} \beta_{i}^{-1} .
\end{aligned}
$$

It fixes $\left(\prod_{i=1}^{g}\left[\alpha_{i}, \beta_{i}\right]\right)$ and so corresponds to an orientation preserving diffeomorphism $\Sigma_{g, 1} \rightarrow \Sigma_{g, 1}$. Let $\iota_{g}: \Sigma_{g, 1} \rightarrow-\Sigma_{g, 1}$ be the composite $-I d \circ \tilde{\iota}_{g}$ where $-\Sigma_{g, 1}$ denotes the surface $\Sigma_{g, 1}$ with opposite orientation.

Lemma 2.1. The oriented manifold $\mathcal{H}_{g} \amalg_{\iota_{g}}-\mathcal{H}_{g}$ obtained by identifying the boundaries of $\mathcal{H}_{g}$ and $-\mathcal{H}_{g}$ via $\iota_{g}$ is diffeomorphic to $\mathbf{S}^{3}$. Here $-\mathcal{H}_{g}$ denotes $\mathcal{H}_{g}$ with opposite orientation.

Proof. Almost by definition $\tilde{\iota}_{g+1}=\tilde{\iota}_{g} \sharp \tilde{\iota}_{1}$, so $\mathcal{H}_{g} \coprod_{\iota_{g}}-\mathcal{H}_{g}$ is diffeomorphic to the connected sum of $g$ copies of $\mathcal{H}_{1} \coprod_{\iota_{1}}-\mathcal{H}_{1}$. The diffeomorphism $\tilde{\iota}_{1}$ exchanges the meridian $\alpha$ and the parallel $\beta$, and it is well known that this construction yields $\mathbf{S}^{3}$. 


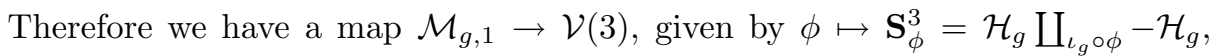
which is compatible with the monomorphisms $\mathcal{M}_{g, 1} \rightarrow \mathcal{M}_{g+1,1}$, thus yielding a well defined and surjective map

$$
\lim _{g \rightarrow+\infty} \mathcal{M}_{g, 1} \longrightarrow \mathcal{V}(3) .
$$

Note that as we identify $\mathbf{S}^{3}$ with $\mathcal{H}_{g} \coprod_{\iota g}-\mathcal{H}_{g}$ the surface $\Sigma_{g}$ becomes the common boundary of the two solid handlebodies $\mathcal{H}_{g}$, and $-\mathcal{H}_{g}$. Denote the image of the restriction maps $\operatorname{Diff}\left(\mathcal{H}_{g}\right.$, rel. $\left.D^{2}\right) \longrightarrow \operatorname{Diff}\left(\Sigma_{g}\right.$, rel. $\left.D^{2}\right)$ and $\operatorname{Diff}\left(-\mathcal{H}_{g}\right.$, rel. $\left.D^{2}\right) \longrightarrow$ $\operatorname{Diff}\left(\Sigma_{g}\right.$, rel. $\left.D^{2}\right)$ in $\mathcal{M}_{g, 1}$, respectively by $\mathcal{B}_{g, 1}$ and $\mathcal{A}_{g, 1}$. It is easily seen that $\mathcal{A}_{g, 1}=\iota_{g}^{-1} \mathcal{B}_{g, 1} \iota_{g}$.

Definition 1. Two mapping classes $\phi, \psi \in \mathcal{M}_{g, 1}$ will be called equivalent, denoted by $\phi \approx \psi$, if there exists $f_{a} \in \mathcal{A}_{g, 1}$ and $f_{b} \in \mathcal{B}_{g, 1}$ such that $\phi=f_{a} \circ \psi \circ f_{b}$. This is equivalent to saying that $\phi$ and $\psi$ lie in the same double coset in $\mathcal{A}_{g, 1} \backslash \mathcal{M}_{g, 1} / \mathcal{B}_{g, 1}$.

The relation $\approx$ is clearly an equivalence relation. It is a classical fact that if $\phi \approx \psi$, then $\mathbf{S}_{\phi}^{3}$ is diffeomorphic to $\mathbf{S}_{\psi}^{3}$ (cf. [7] chap. 8). Moreover two equivalent maps in $\mathcal{M}_{g, 1}$ are also equivalent when considered as maps in $\mathcal{M}_{g+1,1}$. The key result in the theory of Heegaard splittings is:

Theorem 2.2. The map

$$
\begin{aligned}
\lim _{g \rightarrow+\infty} \mathcal{M}_{g, 1} / \approx & \longrightarrow \mathcal{V}(3), \\
\phi & \longmapsto \mathcal{S}_{\phi}^{3}
\end{aligned}
$$

is bijective.

For historical proofs of this theorem we refer the reader to Redemeister [18] or Singer [19]. For a shorter proof see for instance Craggs [2].

Using the Mayer-Vietoris sequence one can prove that the image of the restriction of this map to the equivalence classes of maps in $\mathcal{M}_{g, 1}(1)$ is precisely $\mathcal{S}(3)$. This result was refined by S. Morita who proved:

Theorem 2.3 ([11]). The map

$$
\lim _{g \rightarrow+\infty} \mathcal{M}_{g, 1}(2) / \approx \longrightarrow \mathcal{S}(3)
$$

is a bijection.

Remark 2.1. In the above theorem $\mathcal{M}_{g, 1}(2) / \approx$ stands for the equivalence classes under the equivalence relation $\approx$ restricted to $\mathcal{M}_{g, 1}(2)$. In particular if $\phi \in \mathcal{M}_{g, 1}(2)$ and $\psi \in \mathcal{M}_{g, 1}(2)$ are equivalent, that is, $\phi=f_{a} \circ \psi \circ f_{b}$ for some $\left(f_{a}, f_{b}\right) \in \mathcal{A}_{g, 1} \times \mathcal{B}_{g, 1}$, we do not require $f_{a}$ or $f_{b}$ to belong to $\mathcal{M}_{g, 1}(2)$.

Our aim is to prove that the theorem still holds when we replace $\mathcal{M}_{g, 1}(2)$ by $\mathcal{M}_{g, 1}(3)$, to do so we first need to enrich the structure of the Johnson homomorphisms.

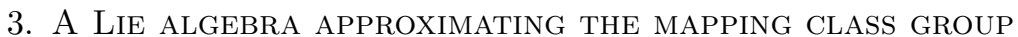

Both graded groups $\bigoplus_{k \geq 1} \mathcal{M}_{g, 1}(k)$ and $\bigoplus_{k \geq 1} \operatorname{Hom}\left(N_{1}, \mathcal{L}_{k+1}\right)$ have natural structures of graded Lie algebras; that these structures are compatible with the Johnson homomorphism was first observed by S. Morita in [12]. Let us briefly recall the involved structures; for the details we refer the reader to [12]. 
The Lie algebra structure on $\bigoplus_{k>1} \mathcal{M}_{g, 1}(k)$ is induced by the commutator bracket $f, g \mapsto[f, g]=f \circ g \circ f^{-1} \circ g^{-1}$, and it is clearly compatible with the action by $\mathcal{M}_{g, 1}$.

The algebra $\bigoplus_{k>1} \mathcal{L}_{k+1}$ is the graded subalgebra of elements of degree $k \geq 2$ in the free graded Lie algebra $\mathcal{L}$ generated by $N_{1}$. Recall that a derivation of a Lie algebra $L$ is a homomorphism of abelian groups $d: L \rightarrow L$, such that for any $u, v \in L, d([u, v])=[d(u), v]+[u, d(v)]$. A derivation of a graded Lie algebra is graded of degree $s$ if for all $k \geq 0, d\left(L_{k}\right) \subset L_{k+s}$. In particular, the abelian group of graded derivations of degree $\geq 1$ of $\mathcal{L}$ is isomorphic to $\bigoplus_{k>1} \operatorname{Hom}\left(N_{1}, \mathcal{L}_{k+1}\right)$ (note that by definition an element $f \in \operatorname{Hom}\left(N_{1}, \mathcal{L}_{k+1}\right)$ is of degree $k$ ). There is a natural graded product on the group of graded derivations:

Definition 2. Let $f$ and $g$ be two derivations of degree $k$ and $l$; then $f\{g\}$ is the unique derivation of degree $k+l$ given by $f\{g\}(u)=f(g(u))$ for all $u \in N_{1}$.

The anti-symmetrisation of this product $[f, g]=f\{g\}-g\{f\}$ induces a Lie bracket, and in our case it is compatible with the action of $\mathcal{M}_{g, 1}$, as was proved by S. Morita.

Recall that we have a symplectic form $\omega: \Lambda^{2} N_{1} \rightarrow \mathbf{Z}$; as usual this form induces an isomorphism of $\mathrm{Sp} \omega$-modules

$$
\begin{aligned}
N_{1} & \rightarrow \operatorname{Hom}\left(N_{1}, \mathbf{Z}\right), \\
a & \mapsto(b \sim \omega(a, b)) .
\end{aligned}
$$

This in turn induces isomorphisms of $\operatorname{Sp} \omega$-modules $\operatorname{Hom}\left(N_{1}, \mathcal{L}_{k+1}\right) \simeq N_{1} \otimes \mathcal{L}_{k+1}$. Let $D_{k}\left(N_{1}\right)$ be the kernel of the Lie bracket $N_{1} \otimes \mathcal{L}_{k+1} \stackrel{[,]}{\longrightarrow} \mathcal{L}_{k+2}$. Denote by $\theta=$ $\sum_{i=1}^{g} a_{i} \wedge b_{i} \in \mathcal{L}_{2}$ the class of $\prod_{i=1}^{g}\left[\alpha_{i}, \beta_{i}\right]$. A typical derivation $\phi \in \operatorname{Hom}\left(N_{1}, \mathcal{L}_{k}\right)$, such that $\phi\left(a_{i}\right)=l_{a_{i}}$ and $\phi\left(b_{i}\right)=l_{b_{i}}$, sends $\theta$ to $\phi(\theta)=\sum_{i=1}^{g}\left[l_{a_{i}}, b_{i}\right]+\left[a_{i}, l_{b_{i}}\right]$. In particular $D\left(N_{1}\right)=\bigoplus_{k=1}^{\infty} D_{k}\left(N_{1}\right)$ is the sub-Lie algebra of $\bigoplus_{k \geq 1} \operatorname{Hom}\left(N_{1}, \mathcal{L}_{k+1}\right)$ of elements that send $\theta$ to 0 . As $\mathcal{M}_{g, 1}$ fixes $\prod_{i=1}^{g}\left[\alpha_{i}, \beta_{i}\right]$, the images of the Johnson homomorphisms lie in $D\left(N_{1}\right)$.

Theorem 3.1 ([12]). The Johnson homomorphisms reassemble into a graded monomorphism of graded Lie algebras and $\mathcal{M}_{g, 1}$-modules:

$$
\bigoplus_{k \geq 1} \tau_{k}: \bigoplus_{k \geq 1} \mathcal{M}_{g, 1}(k) / \mathcal{M}_{g, 1}(k+1) \longrightarrow \bigoplus_{k \geq 1} D_{k}\left(N_{1}\right)
$$

Moreover, the two structures are mutually compatible.

By definition of $D\left(N_{1}\right)$ the action of $\mathcal{M}_{g, 1}$ factors through $\mathrm{Sp} \omega$ and therefore it is also the case for the action on $\bigoplus_{k \geq 1} \mathcal{M}_{g, 1}(k) / \mathcal{M}_{g, 1}(k+1)$.

3.1. Interpretation by tree Lie algebras. The Lie algebra $D\left(N_{1}\right)$ is still difficult to handle for explicit computations. To overcome this problem we embed it into a Lie algebra generated by uni-trivalent trees, labelled by $N_{1}$. Most of the material in this paragraph is known; we refer the interested reader to Levine's paper [9] for a more detailed investigation of the relationship between $D\left(N_{1}\right)$ and tree Lie algebras. Nevertheless we will give a somewhat extended discussion, as our proof makes full use of the involved structures.

In the sequel a tree will always mean a finite, uni-trivalent tree equipped with a cyclic ordering of its trivalent vertices. Recall that a uni-trivalent tree is a tree whose vertices are all of index one or three. Unless otherwise specified, the trivalent 
vertices of the trees we draw are oriented according to the usual trigonometric orientation of the plane. A rooted tree is a tree with a distinguished univalent vertex called the root and represented by $*$. A labelled tree with labels in the set $E$ is a tree $T$ together with a function $l: V_{1}(T) \rightarrow E$, where $V_{1}(T)$ stands for the set of univalent vertices excepting the root in case $T$ is rooted. The label of the univalent vertex $x$ will be denoted by $l_{x}$.

Definition 3. Let $\mathcal{A}\left(N_{1}\right)$ (resp. $\mathcal{A}^{r}\left(N_{1}\right)$ ) denote the free abelian group generated by the uni-trivalent, oriented (resp. rooted) trees with labels in $N_{1}$, modulo multilinearity with respect to the labels, and the relations IHX, AS and " $T \wedge T=0$ " of Figure 3.

$I H X$

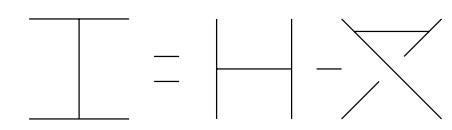

$A S$
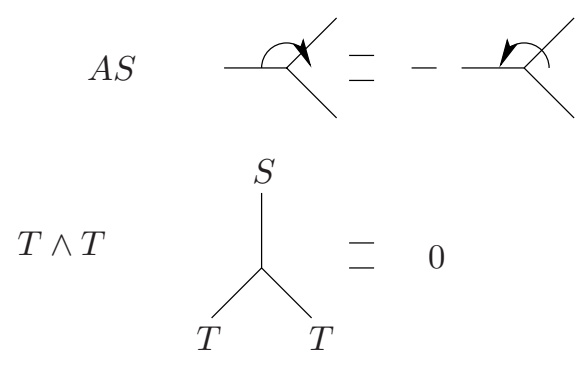

FiguRE 3. Relations among trees

In relation " $T \wedge T=0$ ", $S$ may be either a tree, a label or the root.

Remark 3.1. Usually (cf. [1] [5]) the third relation is omitted, as it is a consequence of the previous one when multiplication by two is an isomorphism on the group of labels, e.g. when $N_{1}$ is replaced by $N_{1} \otimes \mathbf{Q}$.

Both groups $\mathcal{A}^{r}\left(N_{1}\right)$ and $\mathcal{A}\left(N_{1}\right)$ are naturally graded by the internal degree of trees, that is, the number of trivalent vertices in a tree. In the case of $\mathcal{A}\left(N_{1}\right)$, we will only consider the subgroup of elements of degree at least 1 , and still denote it by $\mathcal{A}\left(N_{1}\right)$. The following proposition is well-known:

Proposition 3.1. The bracket in Figure 4 endows $\mathcal{A}^{r}\left(N_{1}\right)$ with a graded Lie algebra

$$
\left(\left.\right|_{A} ^{*},\left.\right|_{B} ^{*}\right)=\wedge_{B}^{*}
$$

FiguRE 4 . Lie bracket in $\mathcal{A}^{r}\left(N_{1}\right)$

structure, compatible with the action of $\mathrm{Sp} \omega . A s \mathcal{A}_{0}^{r}\left(N_{1}\right) \simeq N_{1}$, there is a canonical epimorphism of graded Lie algebras and $\mathrm{Sp} \omega$-modules $\mathcal{L} \longrightarrow \mathcal{A}^{r}\left(N_{1}\right)$, which is in fact an isomorphism. 
In the sequel we identify the graded Lie algebras $\mathcal{L}$ and $\mathcal{A}^{r}\left(N_{1}\right)$. There is a fundamental operation on trees given by gluing two univalent vertices of two distinct trees. Consider two trees $S$ and $T$, where $S$ is not rooted but $T$ may be, and choose two univalent vertices $x \in V_{1}(S)$ and $w \in V_{1}(T)$ (recall that by definition the root * does not belong to $V_{1}(T)$ ). We forget the labelling and glue these two vertices to get a new labelled tree $S-x w-T$, naturally rooted if $T$ was. This gluing operation satisfies:

(1) Symmetry:

$$
S-x w-T=T-w x-S .
$$

(2) Associativity: If at least two of the three trees $S, T, R$ are non-rooted

$$
S-x w-(T-v y-R)=(S-x w-T)-v y-R .
$$

Extending by linearity, this allows one to define:

(1) A bracket on $\mathcal{A}\left(N_{1}\right)$, which is given on trees by:

$$
[S, T]=\sum_{x, y \in V_{1}(S) \times V_{1}(T)} \omega\left(l_{x} \wedge l_{y}\right) S-x y-T .
$$

(2) A natural graded linear action of $\mathcal{A}\left(N_{1}\right)$ on $\mathcal{A}^{r}\left(N_{1}\right)$, which is given on trees by:

$$
S\{T\}=\sum_{x, y \in V_{1}(S) \times V_{1}(T)} \omega\left(l_{x} \wedge l_{y}\right) S-x y-T .
$$

It is readily seen from the definition that this is an action by graded derivations of degree at least 1 . We call the resulting linear map:

$$
\mathcal{A}\left(N_{1}\right) \longrightarrow \bigoplus_{k \geq 1} \operatorname{Hom}\left(N_{1}, \mathcal{L}_{k+1}\right) \simeq N_{1} \otimes \bigoplus_{k \geq 1} \mathcal{L}_{k+1}
$$

the expansion map. On a tree $T \in \mathcal{A}_{k}\left(N_{1}\right)$ the expansion map is given by:

$$
T \longmapsto \sum_{x \in v_{1}(T)} l_{x} \otimes T^{x},
$$

where $T^{x}$ denotes the tree $T$ rooted by its vertex $x$.

In fact more is true, namely the above bracket is a Lie bracket and turns the expansion map into a Lie algebra homomorphism. This was first observed by Garoufalidis and Levine [3] for trees labelled by $N_{1} \otimes \mathbf{Q}$. The proof is straightforward; for the details we refer the interested reader to [6].

Theorem 3.2. - The bracket on $\mathcal{A}\left(N_{1}\right)$ induces a graded Lie algebra structure compatible with the action of $\mathrm{Sp} \omega$ on the labels.

- The expansion map induces a graded homomorphism of Lie algebras and $\mathrm{Sp} \omega$-modules. Its image is contained in $D\left(N_{1}\right)$.

In degree 1 the expansion map is an isomorphism, the group $D_{1}\left(N_{1}\right)$ is isomorphic to $\Lambda^{3} N_{1}$, and the expansion map is given by:

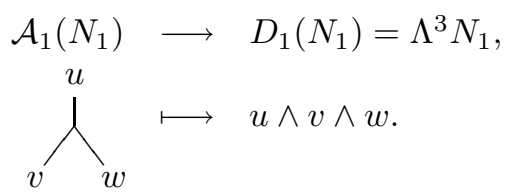

In higher degrees the expansion map is no longer an isomorphism. Nevertheless there is still a linear homomorphism $D\left(N_{1}\right) \rightarrow \mathcal{A}\left(N_{1}\right)$ which is an inverse after taking tensor product with the rationals. It is compatible with the $\mathrm{Sp} \omega$-action but not with the Lie bracket. 
Recall that for each $k \geq 1 \operatorname{Hom}\left(N_{1}, \mathcal{L}_{k+1}\right) \simeq N_{1} \otimes \mathcal{L}_{k+1}$ as an Sp $\omega$-module. Moreover we have identified $\mathcal{L}_{k+1}$ with $\mathcal{A}_{k+1}^{r}\left(N_{1}\right)$. Therefore we view $D_{k}\left(N_{1}\right) \subset$ $\operatorname{Hom}\left(N_{1}, \mathcal{L}_{k+1}\right)$ as an $\operatorname{Sp} \omega$-submodule of $N_{1} \otimes \mathcal{A}_{k+1}^{r}\left(N_{1}\right)$. To each elementary tensor $u \otimes T \in N_{1} \otimes \mathcal{A}_{k+1}^{r}\left(N_{1}\right)$ we associate the tree $T_{u} \in \mathcal{A}_{k}\left(N_{1}\right)$ which is obtained by labelling the root of $T$ by $u$. Extending by linearity we get the "labelling map", $L a b: N_{1} \otimes \mathcal{A}_{k+1}^{r}\left(N_{1}\right) \longrightarrow \mathcal{A}_{k}\left(N_{1}\right)$.

Lemma 3.1. (1) For any $k \geq 1$, the composite

$$
\mathcal{A}_{k}\left(N_{1}\right) \stackrel{\text { expan. }}{\longrightarrow} D_{k}\left(N_{1}\right) \stackrel{L a b}{\longrightarrow} \mathcal{A}_{k}\left(N_{1}\right)
$$

is multiplication by $k+2$.

(2) The labelling map Lab: $D_{k}\left(N_{1}\right) \longrightarrow \mathcal{A}_{k}\left(N_{1}\right)$ is injective.

Proof. (1) A direct computation shows that the image of a tree $T \in \mathcal{A}_{k+1}\left(N_{1}\right)$ in $N_{1} \otimes \mathcal{L}_{k+1}$ is $\sum_{x \in V_{1}(T)} l_{x} \otimes T^{x}$ so that the composite $\mathcal{A}_{k}\left(N_{1}\right) \longrightarrow D_{k}\left(N_{1}\right) \longrightarrow$ $\mathcal{A}_{k}\left(N_{1}\right)$ sends $T$ to $(k+2) T$.

(2) Multiplication by $k+2$ certainly induces an isomorphism after tensorisation by the rationals. In [5] it is proved that the expansion map is also an isomorphism after tensorisation by the rationals. It follows that the map $L a b \otimes \mathbf{Q}$ is an isomorphism. Consider the following commutative diagram:

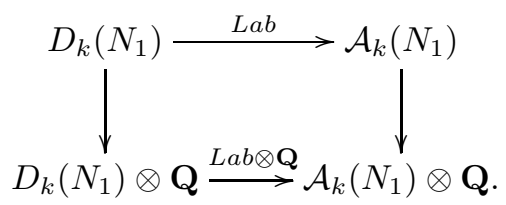

The group $D_{k}\left(N_{1}\right)$ is free abelian, as it is a subgroup of the free abelian group $\operatorname{Hom}\left(N_{1}, \mathcal{L}_{k+1}\right)$. In particular the left vertical arrow is an injection. The bottom arrow is an isomorphism. In particular it is injective. By commutativity of the diagram the composite $D_{k}\left(N_{1}\right) \stackrel{L a b .}{\rightarrow} \mathcal{A}_{k}\left(N_{1}\right) \rightarrow \mathcal{A}_{k}\left(N_{1}\right) \otimes \mathbf{Q}$ is therefore injective, and we may conclude that the labelling map is injective.

\section{The group $\operatorname{Can}\left(\mathcal{A}_{2}\left(N_{1}\right)\right)$}

We focus on the low degree Johnson homomorphisms. In the preceding sections we have constructed homomorphisms that fit into a commutative diagram:

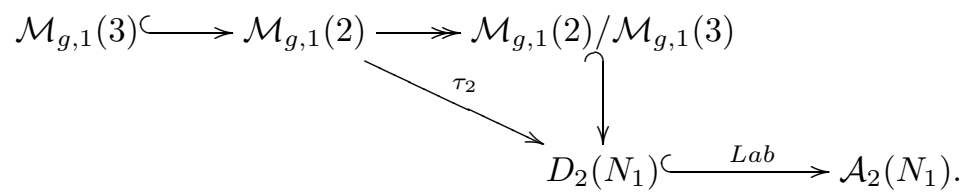

Moreover, all arrows are $\mathcal{M}_{g, 1}$-equivariant, and the action of $\mathcal{M}_{g, 1}$ on the three rightmost groups factors through $\mathrm{Sp} \omega$.

From now on we denote the composite $L a b \circ \tau_{2}$ by $\widetilde{\tau}_{2}$. We denote by $\mathcal{A}_{g, 1}(k)$ (respectively $\mathcal{B}_{g, 1}(k)$ ) the subgroup $\mathcal{A}_{g, 1} \cap \mathcal{M}_{g, 1}(k)$ (respectively $\mathcal{B}_{g, 1} \cap \mathcal{M}_{g, 1}(k)$ ). By definition $\mathcal{A}_{g, 1}(k)$ (resp. $\mathcal{B}_{g, 1}(k)$ ) is normal in $\mathcal{A}_{g, 1}$ (resp. $\mathcal{B}_{g, 1}$ ).

Definition 4. Let $\operatorname{Can}\left(\mathcal{A}_{2}\left(N_{1}\right)\right)$ denote the subgroup of $\mathcal{A}_{2}\left(N_{1}\right)$ generated by $\widetilde{\tau_{2}}\left(\mathcal{A}_{g, 1}(2)\right)$ and $\widetilde{\tau_{2}}\left(\mathcal{B}_{g, 1}(2)\right)$. It is the subgroup of cancelable trees. 
The study of this group is justified by the next proposition.

Proposition 4.1. If $\widetilde{\tau_{2}}\left(\mathcal{M}_{g, 1}(2)\right) \subset \operatorname{Can}\left(\mathcal{A}_{2}\left(N_{1}\right)\right)$, then for any mapping class $\phi \in \mathcal{M}_{g, 1}(2)$ there exists a mapping class $\psi \in \mathcal{M}_{g, 1}(3)$ such that $\phi \approx \psi$ (cf. Definition 1), that is, $S_{\phi}^{3}$ is diffeomorphic to $S_{\psi}^{3}$.

Proof. By definition of $\operatorname{Can}\left(\mathcal{A}_{2}\left(N_{1}\right)\right)$ there exist elements $\phi_{a} \in \mathcal{A}_{g, 1}(2)$ and $\phi_{b} \in$ $\mathcal{B}_{g, 1}(2)$ such that $\widetilde{\tau_{2}}(\phi)=\widetilde{\tau_{2}}\left(\phi_{a}\right)+\widetilde{\tau_{2}}\left(\phi_{b}\right)$. Setting $\psi=\phi_{a}^{-1} \circ \phi \circ \phi_{b}^{-1}$, we have that $\psi \in \mathcal{M}_{g, 1}(3)$ and $\psi \approx \phi$.

To fully exploit this proposition, we need first to have a description of a large class of elements in $\operatorname{Can}\left(\mathcal{A}_{2}\left(N_{1}\right)\right)$ and second to have a description of the image of $\widetilde{\tau}_{2}: \mathcal{M}_{g, 1}(2) \rightarrow \mathcal{A}_{2}\left(N_{1}\right)$.

\subsection{Some families of elements in $\operatorname{Can}\left(\mathcal{A}_{2}\left(N_{1}\right)\right)$.}

4.1.1. Elements arising from bounding simple closed curves. A bounding simple closed curve $\gamma$, BSCC for short, is a simple closed curve such that $\Sigma_{g, 1}-\{\gamma\}$ has two connected components. One of the components of $\Sigma_{g, 1}-\{\gamma\}$, denoted by $\Sigma_{h, \gamma}$, is a surface with only one boundary component, which we may identify with $\gamma$. The genus of $\gamma$ or the genus of the Dehn twist $T_{\gamma}$ is the genus $h$ of $\Sigma_{h, \gamma}$. This terminology was introduced by Johnson, who proved:

Proposition $4.2([8])$. The group $\mathcal{M}_{g, 1}(2)$ is generated by the Dehn twists around $B S C C$.

There is a very nice, algebraic characterisation of the subgroups $\mathcal{A}_{g, 1}$ and $\mathcal{B}_{g, 1}$ due to Griffiths [4]. Choose a map $\phi \in \mathcal{B}_{g, 1}$. Then $\phi$ is the restriction of some diffeomorphism $\Phi: \mathcal{H}_{g} \rightarrow \mathcal{H}_{g}$, and we can assume that $\Phi\left(x_{0}\right)=x_{0}$, where $x_{0}$ is the common base point of $\Sigma_{g, 1}$ and $\mathcal{H}_{g}$. The inclusion $\Sigma_{g, 1} \subset \mathcal{H}_{g}$ induces a surjective homomorphism $\pi_{1}\left(\Sigma_{g, 1}, x_{0}\right) \rightarrow \pi_{1}\left(\mathcal{H}_{g}, x_{0}\right)$, and its kernel is the normal subgroup generated by the set of curves $\left\{\beta_{i}\right\}_{1 \leq i \leq g}$. As $\phi$ is the restriction of $\Phi, \pi_{1}(\phi)$ induces a morphism on $\pi_{1}\left(\mathcal{H}_{g}, x_{0}\right)$, in fact it induces precisely $\pi_{1}(\Phi)$. Therefore, $\pi_{1}(\phi)$ preserves the kernel of $\pi_{1}\left(\Sigma_{g, 1}, x_{0}\right) \rightarrow \pi_{1}\left(\mathcal{H}_{g}, x_{0}\right)$. It turns out that this last property characterises the group $\mathcal{B}_{g, 1}$ :

Proposition $4.3([4])$. An element in $\mathcal{M}_{g, 1}$ is in $\mathcal{B}_{g, 1}$ (resp. $\mathcal{A}_{g, 1}$ ), if and only if it preserves the normal subgroup of $\pi_{1}\left(\Sigma_{g, 1}, x_{0}\right)$ generated by the set $\left\{\beta_{i}\right\}_{1 \leq i \leq g}$ (resp. $\left.\left\{\alpha_{i}\right\}_{1 \leq i \leq g}\right)$, which is the kernel of the canonical morphism $\pi_{1}\left(\Sigma_{g, 1}, x_{0}\right) \rightarrow$ $\pi_{1}\left(\mathcal{H}_{g}, x_{0}\right)$ (resp. $\pi_{1}\left(\Sigma_{g, 1}, x_{0}\right) \rightarrow \pi_{1}\left(-\mathcal{H}_{g}, x_{0}\right)$ ).

We are now ready to describe our first family:

Proposition 4.4. Let $\gamma$ be a simple closed curve on $\Sigma_{g, 1}$ which is contractible in $\mathcal{H}_{g}$; then $T_{\gamma}$ belongs to $\mathcal{B}_{g, 1}$. In particular if $\gamma$ is a BSCC, then $\widetilde{\tau_{2}}\left(T_{\gamma}\right)$ belongs to $\operatorname{Can}\left(\mathcal{A}_{2}\left(N_{1}\right)\right)$. Similarly, if $\gamma \in \Sigma_{g, 1}$ is contractible in $-\mathcal{H}_{g}$, then $T_{\gamma} \in \mathcal{A}_{g, 1}$, and if $\gamma$ is moreover a BSCC, then $\widetilde{\tau_{2}}\left(T_{\gamma}\right) \in \operatorname{Can}\left(\mathcal{A}_{2}\left(N_{1}\right)\right)$.

Proof. By Griffiths' result, Proposition 4.3, it is enough to prove that $\forall 1 \leq i \leq g$, $T_{\gamma}\left(\beta_{i}\right) \in \operatorname{ker}\left(\pi_{1}\left(\Sigma_{g, 1}, x_{0}\right) \rightarrow \pi_{1}\left(\mathcal{H}_{g}, x_{0}\right)\right)$. As $\beta_{i}$ belongs to the kernel, it is enough to prove that $T_{\gamma}\left(\beta_{i}\right)$ is homotopic to $\beta_{i}$ in $\mathcal{H}_{g}$. Up to homotopy the curves $\gamma$ and $\beta_{i}$ meet transversally in a finite number of points $x_{1}, \ldots, x_{s}$. For each $1 \leq j \leq s$ consider $\gamma$ as a based loop at $x_{j}$, and choose a based homotopy $H_{j}: S^{1} \times[0,1] \rightarrow \mathcal{H}_{g}$ between $\gamma$ and the constant loop at $x_{j}$. This is possible since $\gamma$ is contractible in 
$\mathcal{H}_{g}$ by assumption. These homotopies reassemble into a homotopy between $T_{\gamma}\left(\beta_{i}\right)$ and $\beta_{i}$.

If $\gamma$ is a BSCC, then according to Johnson, $T_{\gamma}$ also belongs to $\mathcal{M}_{g, 1}(2)$.

Remark 4.1. There is an easy way to produce a BSCC of genus 1 in $\Sigma_{g, 1}$. Consider two simple closed curves $\lambda$ and $\mu$ intersecting in exactly one point on $\Sigma_{g, 1}$. A tubular neighbourhood of the union $\lambda \cup \mu$ is a subsurface of genus 1 in $\Sigma_{g, 1}$, and its boundary $\delta$ is the desired BSCC of genus 1 . If $[\lambda],[\mu] \in N_{1}$ are the homology classes of the curves $\lambda$ and $\mu$, then the image of $T_{\delta}$ in $\mathcal{A}_{2}\left(N_{1}\right)$ is the tree

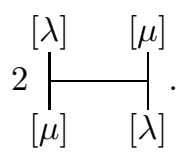

The last assertion follows from a direct computation in the case $\lambda=\alpha_{1}$ and $\mu=\beta_{1}$ together with the fact that $\mathcal{M}_{g, 1}$ acts transitively on the pairs of simple closed curves intersecting exactly in one point.

In the sequel, when producing a BSCC of genus 1, we will only draw the two curves $\lambda$ and $\mu$, and label them by their homology class. The curves $\{\lambda, \mu\}$ are usually called the spine of the sub-surface; as we focus on the boundary we call $\{\lambda, \mu\}$ the spine of the boundary.

4.1.2. Elements arising from commutators. Our second family is more algebraic in nature; it comes from commutators of pairs of elements both sitting in $\mathcal{A}_{g, 1}(1)$ or in $\mathcal{B}_{g, 1}(1)$.

Recall that we have an isomorphism $\mathcal{A}_{1}\left(N_{1}\right) \simeq \Lambda^{3} N_{1}$ induced by the expansion map. According to S. Morita, the images of both $\tau_{1}\left(\mathcal{B}_{g, 1}(1)\right)$ and $\tau_{1}\left(\mathcal{A}_{g, 1}(1)\right)$ have a very simple characterisation in $\Lambda^{3} N_{1} \simeq \mathcal{A}_{1}\left(N_{1}\right)$.

Proposition $4.5([11])$. A tree $\bigwedge_{d}^{c}$ represents an element of $\tau_{1}\left(\mathcal{A}_{g, 1}(1)\right.$ ) (resp. $\left.\mathcal{B}_{g, 1}(1)\right)$ if and only if at least one of its labels belongs to the Lagrangian $L_{A}$ (resp. $\left.L_{B}\right)$.

Corollary 4.1. The group $\widetilde{\tau_{2}}\left(\left[\mathcal{A}_{g, 1}(1), \mathcal{A}_{g, 1}(1)\right]\right) \quad\left(\right.$ resp. $\left.\quad \widetilde{\tau_{2}}\left(\left[\mathcal{B}_{g, 1}(1), \mathcal{B}_{g, 1}(1)\right]\right)\right)$ is generated by the brackets

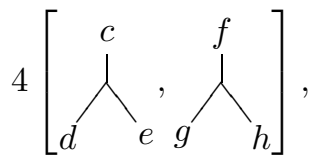

where at least one of the labels $\{c, d, e\}$ and one of the labels $\{f, g, h\}$ are in the Lagrangian $L_{A}$ (resp. $\left.L_{B}\right)$.

Proof. As the Johnson homomorphism defines a Lie algebra homomorphism we have $\tau_{2}\left(\left[\mathcal{B}_{g, 1}(1), \mathcal{B}_{g, 1}(1)\right]\right)=\left[\tau_{1}\left(\mathcal{B}_{g, 1}(1)\right), \tau_{1}\left(\mathcal{B}_{g, 1}(1)\right)\right]$, whence the condition on the labels. This subgroup is obviously contained in $\mathcal{A}_{2}\left(N_{1}\right) \subset D_{2}\left(N_{1}\right)$, and as the composite $\mathcal{A}_{2}\left(N_{1}\right) \hookrightarrow D_{2}\left(N_{1}\right) \rightarrow A_{2}\left(N_{1}\right)$ is multiplication by 4 we get the factor 4 in front of the bracket. 
4.1.3. Invariance properties of $\operatorname{Can}\left(\mathcal{A}_{2}\left(N_{1}\right)\right)$. From the above two families of cancelable trees it is easy to construct more cancelable trees by making use of the elements in $\mathcal{M}_{g, 1}$ that preserve the group $\operatorname{Can}\left(\mathcal{A}_{2}\left(N_{1}\right)\right)$.

In Lemma 2.1 we introduced a mapping class $\iota_{g} \in \mathcal{M}_{g, 1}$ such that $S^{3}=$ $\mathcal{H}_{g} \coprod_{\iota_{g}}-\mathcal{H}_{g}$ and we remarked that $\mathcal{A}_{g, 1}=\iota_{g}^{-1} \mathcal{B}_{g, 1} \iota_{g}$. By equivariance of the map $\widetilde{\tau_{2}}$, the group $\operatorname{Can}\left(\mathcal{A}_{2}\left(N_{1}\right)\right)$ is invariant under the action of $\iota_{g}$.

In the same spirit, if $\phi \in \mathcal{A}_{g, 1}(2)$ and $\Phi \in \mathcal{A}_{g, 1}$, then $\Phi \cdot \widetilde{\tau_{2}}(\phi)=\widetilde{\tau_{2}}\left(\Phi \phi \Phi^{-1}\right) \in$ $\operatorname{Can}\left(\mathcal{A}_{2}\left(N_{1}\right)\right)$, because $\mathcal{A}_{g, 1}(2)$ is normal in $\mathcal{A}_{g, 1}$. The same result holds for $\mathcal{B}_{g, 1}(2)$.

If $T \in \operatorname{Can}\left(\mathcal{A}_{2}\left(N_{1}\right)\right)$ and $\Phi \in \mathcal{A}_{g, 1} \cap \mathcal{B}_{g, 1}$, then $\Phi \cdot T \in \operatorname{Can}\left(\mathcal{A}_{2}\left(N_{1}\right)\right)$. Indeed, by definition of $\operatorname{Can}\left(\mathcal{A}_{2}\left(N_{1}\right)\right)$, we can decompose $T$ into a sum $T=T_{a}+T_{b}$, where $T_{a} \in \widetilde{\tau_{2}}\left(\mathcal{A}_{g, 1}(2)\right)$ and $T_{b} \in \widetilde{\tau_{2}}\left(\mathcal{B}_{g, 1}(2)\right)$ and then apply the preceding remarks to $T_{a}$ and $T_{b}$.

Recall that the action of $\mathcal{M}_{g, 1}$ on $\mathcal{A}_{2}\left(N_{1}\right)$ factors through $\mathrm{Sp} \omega$, so to fully exploit the preceding theorem we need to know the images of $\mathcal{A}_{g, 1}, \mathcal{B}_{g, 1}$ and $\iota_{g}$ in $\operatorname{Sp} \omega$.

The action of $\iota_{g}$ is easy to deduce from its action in homology which is given by: $\iota\left(a_{i}\right)=-b_{i}$ and $\iota\left(b_{i}\right)=a_{i}$, for all $1 \leq i \leq g$.

In [20] Suzuki describes generators for the group $\mathcal{B}_{g, 1}$ and therefore for $\mathcal{A}_{g, 1}=$ $\iota_{g} \mathcal{B}_{g, 1} \iota_{g}^{-1}$. Their action on the basis $\left\{a_{i}, b_{i}\right\}_{1 \leq i \leq g}$ of $N_{1}$ is summarised in the following table (if an element of the basis is omitted, it is fixed under the action). The names of the generators are those given by Suzuki.

\begin{tabular}{|c|c|}
\hline Common generators to $\mathcal{A}_{g, 1}$ and $\mathcal{B}_{g, 1}$ & Action on the basis of $N_{1}$ \\
\hline Twist of the knob $i$ & $a_{i} \rightarrow-a_{i}$ \\
& $b_{i} \rightarrow-b_{i}$ \\
\hline Exchange of knobs $i$ and $j$ & $a_{i} \leftrightarrow a_{j}$ \\
& $b_{i} \leftrightarrow b_{j}$ \\
\hline \hline Generators of $\mathcal{A}_{g, 1}$ alone & Action on the basis of $N_{1}$ \\
\hline A-twist of the $i$ handle & $b_{i} \rightarrow b_{i}-a_{i}$ \\
\hline Sliding $\theta_{A, i, j}$ & $a_{i} \rightarrow a_{i}+a_{j}$ \\
& $b_{j} \rightarrow b_{j}-b_{i}$ \\
\hline Sliding $\xi_{A, i, j}$ & $b_{i} \rightarrow b_{i}-a_{j}$ \\
& $b_{j} \rightarrow b_{j}-a_{i}$ \\
\hline \hline Generators of $\mathcal{B}_{g, 1}$ alone & Action on the basis of $N_{1}$ \\
\hline B-twist of the $i$ handle & $a_{i} \rightarrow a_{i}-b_{i}$ \\
\hline Sliding $\theta_{B, i, j}$ & $a_{i} \rightarrow a_{i}-a_{j}$ \\
& $b_{j} \rightarrow b_{j}+b_{i}$ \\
\hline Sliding $\xi_{B, i, j}$ & $a_{i} \rightarrow a_{i}-b_{j}$ \\
& $a_{j} \rightarrow a_{j}-b_{i}$ \\
\hline
\end{tabular}

It is a standard fact that the subgroup of the elements of $\operatorname{Sp} \omega$ that respect the orthogonal decomposition $N_{1}=L_{A} \oplus L_{B}$ is naturally isomorphic to $\mathrm{GL}_{g}(\mathrm{Z})$. In particular, if $P$ denotes the canonical projection $P: \mathcal{M}_{g, 1} \rightarrow \operatorname{Sp} \omega$, we have $P\left(\mathcal{A}_{g, 1}\right) \cap P\left(\mathcal{B}_{g, 1}\right) \subset G l_{g}(\mathbf{Z})$. The "sliding" and the "twists of the knobs" morphisms hit the standard generators of $G l_{g}(\mathbf{Z})$; therefore:

Lemma 4.1. In $\operatorname{Sp} \omega$ we have $G l_{g}(\mathbf{Z})=P\left(\mathcal{A}_{g, 1}\right) \cap P\left(\mathcal{B}_{g, 1}\right)$.

Summarising, we have:

Theorem 4.1. The group $\operatorname{Can}\left(\mathcal{A}_{2}\left(N_{1}\right)\right)$ is invariant:

(1) Under the action of $\iota_{g}$. 
(2) Under the action of $G l_{g}(\mathbf{Z})=P\left(\mathcal{A}_{g, 1}\right) \cap P\left(\mathcal{B}_{g, 1}\right)$.

(3) If $T \in \operatorname{Can}\left(\mathcal{A}_{2}\left(N_{1}\right)\right)$ is contained in $\widetilde{\tau_{2}}\left(\mathcal{A}_{g, 1}(2)\right)$, then for all $\Phi \in \mathcal{A}_{g, 1}$ $\Phi \cdot T \in \operatorname{Can}\left(\mathcal{A}_{2}\left(N_{1}\right)\right)$.

(4) If $T \in \operatorname{Can}\left(\mathcal{A}_{2}\left(N_{1}\right)\right)$ is contained in $\widetilde{\tau_{2}}\left(\mathcal{B}_{g, 1}(2)\right)$, then for all $\Phi \in \mathcal{B}_{g, 1}$ $\Phi \cdot T \in \operatorname{Can}\left(\mathcal{A}_{2}\left(N_{1}\right)\right)$.

4.2. The image of $\widetilde{\tau_{2}}: \mathcal{M}_{g, 1}(2) \rightarrow \mathcal{A}_{2}\left(N_{1}\right)$. Here is a stronger version of Proposition 4.2, also due to Johnson.

Theorem $4.2([8])$. For $g \geq 3$, the group $\mathcal{M}_{g, 1}(2)$ is generated by the Dehn twists around $B S C C$ of genus 1 and 2 .

The mapping class group acts naturally on the set of simple closed curves, and two BSCC are in the same orbit if and only if they have the same genus. It follows easily from this that two Dehn twists around BSCC are conjugated in $\mathcal{M}_{g, 1}$ if and only if they have the same genus. Therefore $\mathcal{M}_{g, 1}(2)$ is generated by the conjugates of any two Dehn twists $T_{\gamma_{1}}$ and $T_{\gamma_{2}}$ of genus 1 and 2 respectively and $\widetilde{\tau_{2}}\left(\mathcal{M}_{g, 1}(2)\right)$ is generated by the orbits of $\widetilde{\tau_{2}}\left(T_{\gamma_{1}}\right)$ and $\widetilde{\tau_{2}}\left(T_{\gamma_{2}}\right)$ under the action of $\mathcal{M}_{g, 1}$. The algebraic version, in terms of trees, of these results is given by:

Theorem 4.3. The image of $\widetilde{\tau}_{2}$ in $\mathcal{A}_{2}\left(N_{1}\right)$ is the $\mathcal{M}_{g, 1}$-module generated by the elements of the form
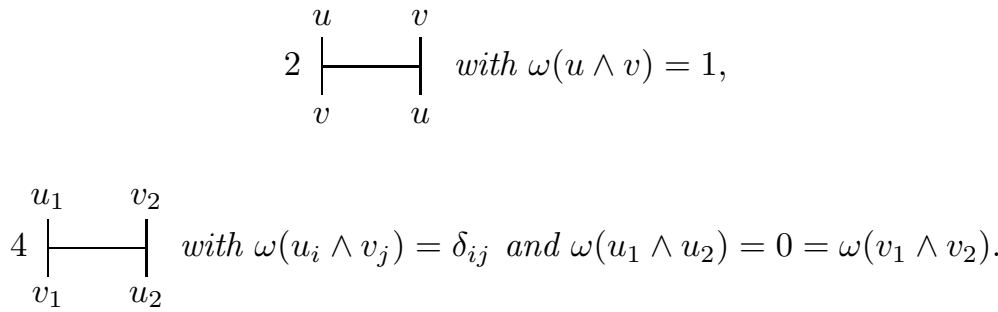

Proof. We choose as curves $\gamma_{1}$ and $\gamma_{2}$ the curves of genus 1 and 2 parallel to the boundary as in Figure 5 .

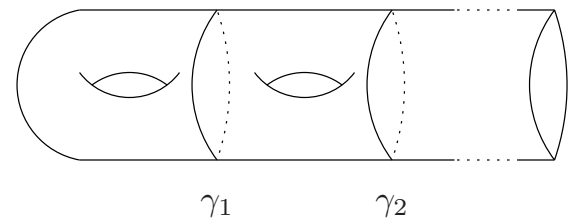

Figure 5. Curves $\gamma_{1}$ and $\gamma_{2}$

A direct computation shows that $T_{\gamma_{1}}$ acts on $\alpha_{1}, \beta_{1} \in \pi_{1}\left(\Sigma_{g, 1}\right)$ as the conjugation by $\left[\alpha_{1}, \beta_{1}\right]$, and leaves the other generators $\alpha_{i}, \beta_{i} 2 \leq i \leq g$ unchanged. By definition of our maps,

$$
\tau_{2}\left(T_{\gamma_{1}}\right)=a_{1} \otimes\left[b_{1},\left[a_{1}, b_{1}\right]\right]+b_{1} \otimes\left[a_{1},\left[b_{1}, a_{1}\right]\right]
$$

and

$$
\widetilde{\tau_{2}}\left(T_{\gamma_{1}}\right)=\left.\left.2\right|_{b_{1}} ^{a_{1}}\right|_{a_{1}} ^{b_{1}} .
$$


The orbit of this last element is clearly the set of trees of the form $\left.\left.2\right|_{v} ^{u}\right|_{u} ^{v}$ such that $\omega(u \wedge v)=1$.

Similarly, writing the action of $T_{\gamma_{2}}$ on curves $\alpha_{1}, \beta_{1}, \alpha_{2}, \beta_{2}$ one gets:

$$
\begin{aligned}
\tau_{2}\left(T_{\gamma_{2}}\right)=b_{1} & \otimes\left[a_{1},\left[b_{1}, a_{1}\right]\right]+b_{1} \otimes\left[a_{1},\left[b_{2}, a_{2}\right]\right] \\
& +a_{1} \otimes\left[b_{1},\left[a_{1}, b_{1}\right]\right]+a_{1} \otimes\left[b_{1},\left[a_{2}, b_{2}\right]\right] \\
& +b_{2} \otimes\left[a_{2},\left[b_{1}, a_{1}\right]\right]+b_{2} \otimes\left[a_{2},\left[b_{2}, a_{2}\right]\right] \\
& +a_{2} \otimes\left[b_{2},\left[a_{1}, b_{1}\right]\right]+a_{2} \otimes\left[b_{2},\left[a_{2}, b_{2}\right]\right],
\end{aligned}
$$

and:

$$
\widetilde{\tau_{2}}\left(T_{\gamma_{2}}\right)=\left.2\right|_{b_{1}} ^{a_{1}}+\left.\left.4\right|_{b_{1}} ^{b_{1}}\right|_{a_{2}} ^{a_{1}}+\left.\left.2\right|_{b_{2}} ^{b_{2}}\right|_{a_{2}} ^{a_{2}} .
$$

The orbit of this element gives, up to elements coming from the orbit of $\widetilde{\tau_{2}}\left(T_{\gamma_{1}}\right)$, all trees of the form $\left.\left.4\right|_{v_{1}} ^{u_{1}}\right|_{u_{2}} ^{v_{2}}$ where $\omega\left(u_{1} \wedge v_{1}\right)=1=\omega\left(u_{2} \wedge v_{2}\right)$ and $\omega\left(u_{1} \wedge u_{2}\right)=$ $\omega\left(u_{1} \wedge v_{2}\right)=0=\omega\left(u_{2} \wedge v_{1}\right)$.

\section{Proof of the Main Theorem}

Main Theorem. Any integral homology sphere is diffeomorphic to $S_{\phi}^{3}$ for some $\phi \in \mathcal{M}_{g, 1}(3)$ with $g \geq 9$.

By Morita's result, we have a bijection (cf. Theorem 2.3):

$$
\lim _{g \rightarrow+\infty} \mathcal{M}_{g, 1}(2) / \approx \longrightarrow \mathcal{S}(3) .
$$
tion:

Thus it suffices to show that the inclusions $\mathcal{M}_{g, 1}(3) \hookrightarrow \mathcal{M}_{g, 1}(2)$ induce a bijec-

$$
\lim _{g \rightarrow+\infty} \mathcal{M}_{g, 1}(3) / \approx \longrightarrow \lim _{g \rightarrow+\infty} \mathcal{M}_{g, 1}(2) / \approx
$$

This is the case if for large enough values of $g$ any element in $\mathcal{M}_{g, 1}(2)$ is equivalent to some element in $\mathcal{M}_{g, 1}(3)$. By Proposition 4.1 this will follow from:

Theorem 5.1. For $g \geq 9$, the image of $\widetilde{\tau_{2}}: \mathcal{M}_{g, 1}(2) \rightarrow \mathcal{A}_{2}\left(N_{1}\right)$ belongs to the subgroup $\operatorname{Can}\left(\mathcal{A}_{2}\left(N_{1}\right)\right)$.

5.1. Three technical lemmas. In the sequel we will work "modulo the subgroup $\operatorname{Can}\left(\mathcal{A}_{2}\left(N_{1}\right)\right)$ ". Two elements, say $S, T$ in $\mathcal{A}_{2}\left(N_{1}\right)$, which differ by an element of $\operatorname{Can}\left(\mathcal{A}_{2}\left(N_{1}\right)\right)$ will be called equivalent, denoted by $S \approx T$. An element equivalent to 0 will be called cancelable. It is obvious that being equivalent is invariant under the action of $\mathrm{GL}_{g}(\mathbf{Z})$. We also assume that $g \geq 9$.

Lemma 5.1. For all $i \neq j$ the following trees are in $\tilde{\tau_{2}}\left(\mathcal{A}_{g, 1}(2)\right) \cap \tilde{\tau_{2}}\left(\mathcal{B}_{g, 1}(2)\right) \subset$ $\operatorname{Can}\left(\mathcal{A}_{2}\left(N_{1}\right)\right)$ :

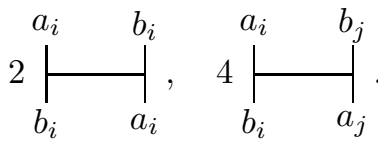


Proof. The trees $\left.\left.2\right|_{b_{i}} ^{a_{i}}\right|_{a_{i}} ^{b_{i}}$ are all in the $\mathrm{GL}_{g}(\mathbf{Z})$-orbit of $\left.2\right|_{b_{1}} ^{a_{1}} a_{a_{1}}^{b_{1}}$, which is cancelable since the curve $\gamma_{1}$ in Figure 5 is contractible in both $\mathcal{H}_{g}$ and $-\mathcal{H}_{g}$. By Propsition 4.4 they are all cancelable. The same argument holds for $\left.4\right|_{b_{i}} ^{a_{i}} a_{j}$ since, up to trees of the preceding form, it belongs to the $\mathrm{GL}_{g}(\mathbf{Z})$-orbit of the curve $\gamma_{2}$, which is contractible, too.

Column Intersection Lemma 5.2. Suppose that the tree $\left.\left.4\right|_{d} ^{c}\right|_{e} ^{f}$ is labelled by elements of the symplectic basis $\left\{a_{i}, b_{i}\right\}_{1 \leq i \leq g}$. Assume furthermore that $\omega(c \wedge e)=$ $\omega(c \wedge f)=0=\omega(d \wedge e)=\omega(d \wedge f)$. Then $\left.\left.4\right|_{d} ^{c}\right|_{e} ^{f}$ is cancelable.

Proof. As $g \geq 9$, there is some index $j$ such that the sets $\left\{a_{j}, b_{j}\right\}$ and $\{c, d, e, f\}$ are disjoint. Assume $c \in\left\{b_{i}\right\}_{1 \leq i \leq g}$. According to Proposition 4.5 both elements $\bigwedge_{c}^{a_{j}}$ and $\bigwedge_{f}^{b_{j}}$ represent elements of $\tau_{1}\left(\mathcal{B}_{g, 1}(1)\right)$. By definition of the bracket:

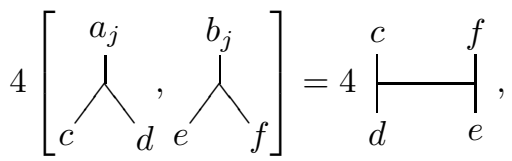

which is cancelable by Corollary 4.1 .

If $c \in\left\{a_{i}\right\}_{1 \leq i \leq g}$, just replace the pair $\left(a_{j}, b_{j}\right)$ in the last equality by $\left(-b_{j}, a_{j}\right)$.

Chain Equivalences Lemma 5.3. For all pairwise distinct indices $i, j, k$, we have three chain of equivalences:

1)

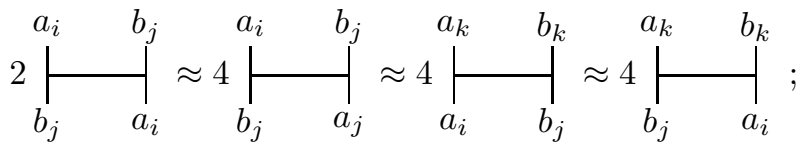

2)

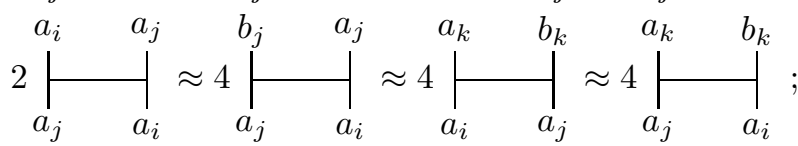

3)

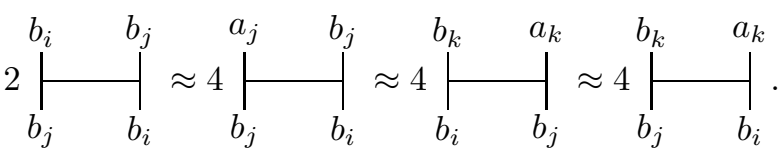

Moreover, any of these elements is equivalent to its opposite, so that 2 times any of these elements is cancelable.

Proof. Using a suitable exchange of knobs we may suppose that $i=1, j=2$ and $k=3$. 


\section{First chain of equivalences}

Observe that the curve $\delta$, whose spine is drawn in Figure 6, is contractible in $\mathcal{H}_{g}$, in particular $\widetilde{\tau_{2}}\left(T_{\delta}\right)$ is cancelable. Now,

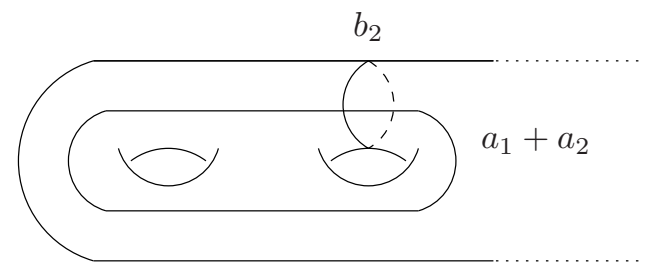

Figure 6. Spine of the curve $\delta$

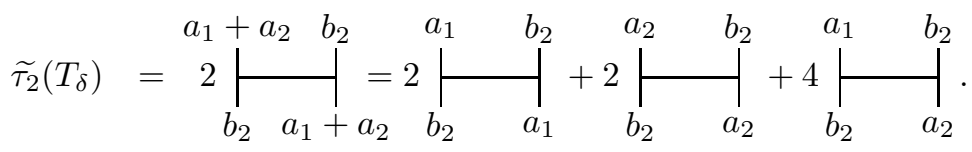

As $\left.2\right|_{b_{2}} ^{a_{2}} a_{2}$ is cancelable, we get $\left.\left.2\right|_{b_{2}} ^{b_{2}}\right|_{a_{1}} ^{a_{1}} \approx-\left.\left.4\right|_{b_{2}} ^{b_{2}}\right|_{a_{2}} ^{a_{1}}$. Applying the "twist of the knob 2 " to both sides removes the minus sign.

The equality

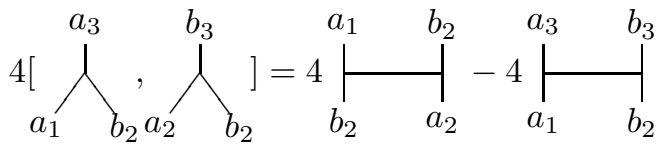

shows that $\left.\left.\left.\left.4\right|_{b_{2}} ^{a_{1}}\right|_{a_{2}} ^{b_{2}} \approx 4\right|_{a_{1}} ^{a_{3}}\right|_{b_{2}} ^{b_{3}}$. Exchanging the roles of $a_{3}$ and $b_{3}$ in the previous bracket we get $\left.4\right|_{b_{2}} ^{a_{1}} \approx-\left.4\right|_{a_{2}} ^{b_{2}} \approx b_{2}$.

Notice that we proved that $\left.\left.\left.4\right|_{b_{2}} ^{a_{1}} \approx 2\right|_{a_{2}} ^{b_{2}} \approx\right|_{a_{2}} ^{a_{1}} \approx-\left.\left.4\right|_{b_{2}} ^{b_{2}}\right|_{a_{2}} ^{a_{1}}$. In particular $\left.\left.\left.\left.4\right|_{b_{2}} ^{a_{1}}\right|_{a_{1}} ^{b_{2}} \approx 4\right|_{b_{2}} ^{a_{1}}\right|_{a_{2}} ^{b_{2}}-\left.4\right|_{b_{2}} ^{a_{1}}=0$. This proves that any tree in this chain is equivalent to its opposite, and therefore that two times any tree involved in this chain of equivalences is cancelable. A similar argument works for the other two chains of equivalences.

\section{Second and third chains of equivalences}

The second chain of equivalences is proved in the same way as the first one. One has just to replace the curve $\delta$ by the curve $\delta^{\prime}$ whose spine is drawn in Figure 7 and which is contractible in $-\mathcal{H}_{g}$. The third chain of equivalences is obtained from the 


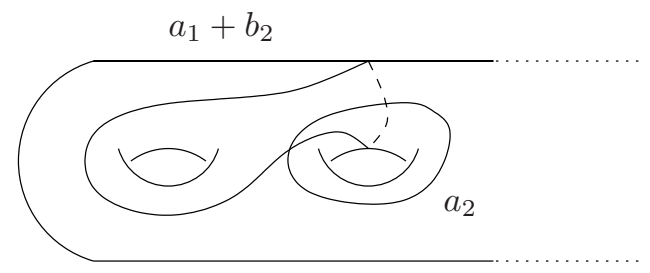

Figure 7. Spine of the curve $\delta^{\prime}$

second one by applying $\iota_{g}$ termwise.

5.2. Proof of Theorem 5.1. We are now in a position to prove that the image of $\widetilde{\tau_{2}}$ belongs to $\operatorname{Can}\left(\mathcal{A}_{2}\left(N_{1}\right)\right)$. It is obviously enough to prove that the image of the generators of $\mathcal{M}_{g, 1}(2)$, that is, Dehn twists of genus 1 and 2, belong to $\operatorname{Can}\left(\mathcal{A}_{2}\left(N_{1}\right)\right)$.

The proof of Theorem 5.1 is quite straightforward once we have in hand the three above technical Lemmas 5.1, 5.2 and 5.3, but nevertheless it is a bit long and proceeds by "case by case" computations. Therefore we will only write the main lines of the proof, leaving the details of the computations to the interested reader.

5.2.1. Images of genus 1 twists are in $\operatorname{Can}\left(\mathcal{A}_{2}\left(N_{1}\right)\right)$. By the description of the images of genus 1 BSCC (see Theorem 4.3) it is enough to prove

Theorem 5.2. Assume $g \geq 9$. For any pair of elements $u, w \in N_{1}$ such that $\omega(u \wedge w)=1$,

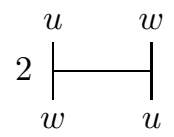

is cancelable.

If $x, y \in N_{1}$ we denote by $\langle x, y\rangle \subset N_{1}$ the submodule they generate.

Proof. First step: Without loss of generality we may suppose that $u, w \in$ $\left\langle a_{1}, a_{2}, b_{1}, b_{2}\right\rangle$.

Recall that $N_{1}=L_{A} \oplus L_{B}$ and decompose $u$ and $w$ as $u=a_{u}+b_{u}$ and $w=$ $a_{w}+b_{w}$. The elements $a_{u}$ and $a_{w}$ are contained in a direct factor of $A$ of rank at most 2 ; therefore there exists $\phi \in \mathrm{GL}_{g}(\mathbf{Z})$ such that $\phi\left(a_{u}\right)$ and $\phi\left(a_{w}\right)$ belong to $\left\langle a_{1}, a_{2}\right\rangle$. Similarly $\phi\left(b_{u}\right)$ and $\phi\left(b_{w}\right)$ are the sum of elements contained in $\left\langle b_{1}, b_{2}\right\rangle$ and of two elements $\hat{b}_{u}$ and $\hat{b}_{w}$ contained in $\left\langle b_{3}, \ldots, b_{g}\right\rangle$. As $\hat{b}_{u}$ and $\hat{b}_{w}$ are contained in a direct factor of $\left\langle b_{3}, \ldots, b_{g}\right\rangle$ of rank at most two, there exists $\psi \in \mathrm{GL}_{g}(\mathbf{Z})$ such that $\left.\psi\right|_{\left\langle b_{1}, b_{2}\right\rangle}=I d=\left.{ }^{t} \psi^{-1}\right|_{\left\langle a_{1}, a_{2}\right\rangle}$ and $\psi\left(\left\langle\hat{b}_{u}, \hat{b}_{w}\right\rangle\right) \subset\left\langle b_{3}, b_{4}\right\rangle$. By construction the labels of the trees

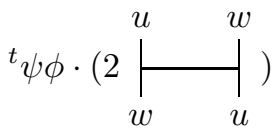


are contained in the desired submodule. As a consequence of Theorem 4.1, $\left.2 \underset{w}{u}\right|_{u} ^{w}$ is cancelable if and only if ${ }^{t} \psi \phi\left(\left.2 \underset{w}{u}\right|_{u} ^{u}\right)$ is cancelable.

As an intermediary step, we may thus assume that $u, w \in\left\langle a_{1}, a_{2}, b_{1}, b_{2}, b_{3}, b_{4}\right\rangle$. By symmetry, to reduce the module to $\left\langle a_{1}, a_{2}, b_{1}, b_{2}\right\rangle$ it is enough to prove that if $w=w^{\prime}+\hat{b}_{w}$ with $w^{\prime} \in\left\langle a_{1}, a_{2}, b_{1}, b_{2}\right\rangle$ and $\hat{b}_{w} \in\left\langle b_{3}, b_{4}\right\rangle$, then

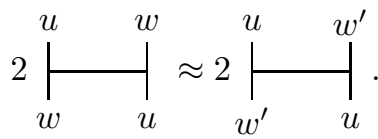

By multilinearity of the labels:

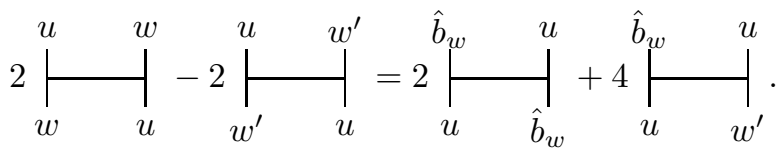

Computing the cancelable bracket

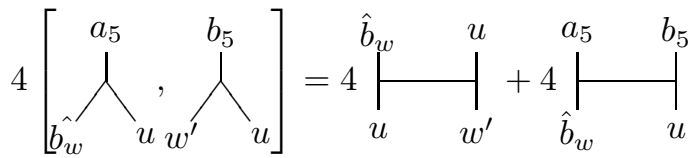

we see that

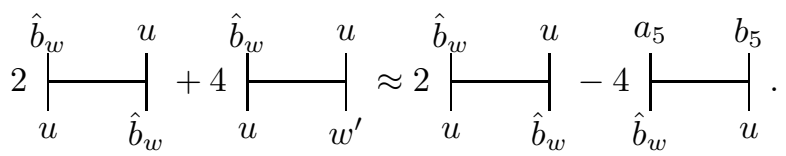

We have to prove that the right-hand term is cancelable.

Write $u=n_{1} a_{1}+n_{2} a_{2}+\sum_{i=1}^{4} m_{i} b_{i}$ and expand $\left.2\right|_{u} ^{\hat{b}_{w}} \hat{b}_{w}-\left.4 \hat{b}_{w}\right|_{u} ^{a_{5}}$ by multilinearity. It is equal to

$$
\begin{aligned}
& \sum_{i=1}^{2}\left(\left.2 n_{i}^{2}\right|_{a_{i}} ^{\hat{b}_{w}} \hat{b}_{w}^{a_{i}}-4 n_{i} \hat{b}_{w}^{a_{5}} \hat{a}_{i}\right)+\sum_{i=1}^{b_{5}}\left(\left.2 m_{i}^{2}\right|_{b_{i}} ^{\hat{b}_{w}} \hat{b}_{w}-4 m_{i} \hat{b}_{w} b_{i} b_{i}\right) \\
& +\left.\sum_{1 \leq i \neq j \leq 4} 4 n_{i} m_{j}\right|_{a_{i}} ^{\hat{b}_{w}} \hat{b}_{w} .
\end{aligned}
$$

By the Column Intersection Lemma 5.2, each term in the third sum is cancelable. The terms in two remaining sums are handled in the same way. We only write the details for terms in the first sum. 
Lemma 5.4. For $1 \leq i \leq 2$, the sum,

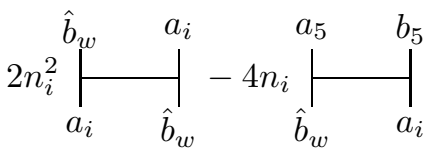

is cancelable.

Proof. Write $\hat{b}_{w}=s b_{3}+t b_{4}$. Expanding by multilinearity one finds that

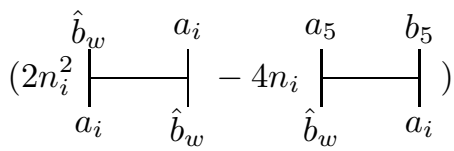

is equivalent to:

$$
\left.\left.4\left(\left(n_{i} s\right)^{2}-n_{i} s\right)\right|_{b_{3}} ^{a_{5}}\right|_{a_{i}} ^{b_{5}}+\left.\left.4\left(\left(n_{i} t\right)^{2}-n_{i} t\right)\right|_{b_{4}} ^{a_{5}}\right|_{a_{i}} ^{b_{5}} .
$$

We have proved in the Chain Equivalences Lemma 5.3 that the 2 times any term that appears in a "chain equivalence" is cancelable. As $\left.4\right|_{b_{3}} ^{a_{5}} b_{i}$ and $\left.4\right|_{b_{4}} ^{b_{5}} a_{i}$ both appear in the first chain and both $\left(\left(n_{i} s\right)^{2}-n_{i} s\right)$ and $\left(\left(n_{i} t\right)^{2}-n_{i} t\right)$ are even, the sum is cancelable.

Second step: Assume that $u, w \in\left\langle a_{1}, a_{2}, b_{1}, b_{2}\right\rangle$. Set $u=u_{1}^{a} a_{1}+u_{2}^{a} a_{2}+u_{1}^{b} b_{1}+u_{2}^{b} b_{2}$ and $w=w_{1}^{a} a_{1}+w_{2}^{a} a_{2}+w_{1}^{b} b_{1}+w_{2}^{b} b_{2}$.

We expand the tree $\left.\left.2\right|_{w} ^{u}\right|_{u} ^{w}$ by multilinearity and group the trees by type, according to the number of $a_{i}$ 's and $b_{i}$ 's that appear as labels. The types are thus $(4 a),(3 a, 1 b)(2 a, 2 b),(1 a, 3 b)$ and $(4 b)$. We will inspect these trees by type and show that after grouping equivalent trees, they are all cancelable.

The coefficient of the tree $\left.\left.\right|_{y_{j}} ^{x_{i}}\right|_{z_{k}} ^{t_{l}}$ is $\left(u_{i}^{x} w_{j}^{y}-u_{j}^{y} w_{i}^{x}\right)\left(u_{k}^{z} w_{l}^{t}-u_{l}^{t} w_{k}^{z}\right)$ up to a coefficient 2 or 4 depending on the symmetry of the tree.

Types (4a) and (3a,1b)

\begin{tabular}{|c|c|c|c|}
\hline \multirow{3}{*}{ Tree } & $a_{1}$ & $a_{1}$ & $a_{1}$ \\
\hline & 2 & 4 & 4 \\
\hline & $a_{1}$ & $a_{1}$ & $a_{2}$ \\
\hline Coefficient & $\begin{array}{c}\left(u_{1}^{a} w_{2}^{a}-u_{2}^{a} w_{1}^{a}\right) \times \\
\left(u_{1}^{a} w_{2}^{a}-u_{2}^{a} w_{1}^{a}\right) \\
\end{array}$ & $\begin{array}{c}\left(u_{1}^{a} w_{2}^{a}-u_{2}^{a} w_{1}^{a}\right) \times \\
\left(u_{1}^{a} w_{1}^{b}-u_{1}^{b} w_{1}^{a}\right) \\
\end{array}$ & $\begin{array}{c}\left(u_{1}^{a} w_{2}^{a}-u_{2}^{a} w_{1}^{a}\right) \times \\
\left(u_{2}^{a} w_{2}^{b}-u_{2}^{b} w_{2}^{a}\right) \\
\end{array}$ \\
\hline
\end{tabular}

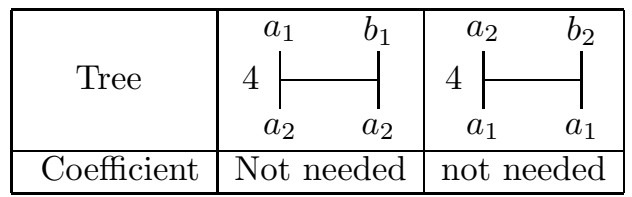


By the second chain of equivalences the first 3 trees are mutually equivalent. As in Lemma 5.4, to prove that their sum is cancelable it is enough to prove that the sum of their coefficients is even. A direct computation using the fact that $\omega(u \wedge v)=1$ yields the result.

The last two trees are in the same $G l_{g}(\mathbf{Z})$-orbit, therefore one is cancelable if and only if the other is cancelable. The proof of the cancellation of the tree $4 \underset{a_{2}}{a_{2}}$ is obtained by applying the Chain Equivalences Lemma 5.3 to the trees that appear from the spine of the curve $\delta^{\prime \prime}$ on Figure 8.

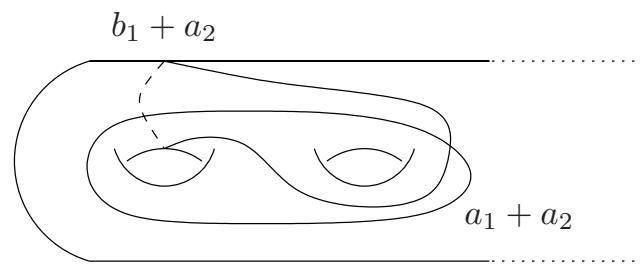

FiguRE 8. Spine of $\delta^{\prime \prime}$

\section{Type (4b) and $(3 a, 1 b)$}

These trees are obtained from the preceding list by interchanging the roles of $a$ and $b$. The same argument as above shows that their sum is cancelable.

\section{Type $(2 \mathrm{a}, 2 \mathrm{~b})$}

There are 11 trees of this type to consider. Among them, 3 are cancelable by Lemma 5.1:

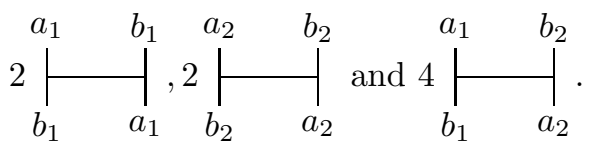

For the remaining trees, as above, we have two groups of 3 trees that are handled by the Chain Equivalences Lemma, and two that need a particular argument to be cancelled. The two groups of 3 trees are:

\begin{tabular}{|c|c|c|c|}
\hline & $a_{2}$ & $a_{2}$ & $a_{2}$ \\
\hline Tree & $4 \underset{b_{1}}{a_{1}}$ & $2 \underset{b_{1}}{a_{2}}$ & $4 \underset{b_{1}}{\longmapsto}$ \\
\hline Coefficient & $\begin{array}{c}\left(u_{2}^{a} w_{1}^{b}-u_{1}^{b} w_{2}^{a}\right) \times \\
\left(u_{1}^{a} w_{1}^{b}-u_{1}^{b} w_{1}^{a}\right)\end{array}$ & $\begin{array}{c}\left(u_{2}^{a} w_{1}^{b}-u_{1}^{b} w_{2}^{a}\right) \times \\
\left(u_{2}^{a} w_{1}^{b}-u_{1}^{b} w_{2}^{a}\right)\end{array}$ & $\begin{array}{c}\left(u_{2}^{a} w_{1}^{b}-u_{1}^{b} w_{2}^{a}\right) \times \\
\left(u_{2}^{a} w_{2}^{b}-u_{2}^{b} w_{2}^{a}\right)\end{array}$ \\
\hline
\end{tabular}

\begin{tabular}{|c|c|c|c|}
\hline & $a_{1}$ & $a_{1}$ & $a_{1}$ \\
\hline Tree & $4 \prod_{b_{2}}$ & $2 \underset{b_{2}}{2}$ & $4 \underset{b_{1}}{a_{1}}$ \\
\hline Coefficient & $\begin{array}{c}\left(u_{1}^{a} w_{2}^{b}-u_{2}^{b} w_{1}^{a}\right) \times \\
\left(u_{2}^{a} w_{2}^{b}-u_{2}^{b} w_{2}^{a}\right)\end{array}$ & $\begin{array}{c}\left(u_{1}^{a} w_{2}^{b}-u_{2}^{b} w_{1}^{a}\right) \times \\
\left(u_{1}^{a} w_{2}^{b}-u_{2}^{b} w_{1}^{a}\right)\end{array}$ & $\begin{array}{c}\left(u_{1}^{a} w_{1}^{b}-u_{1}^{b} w_{1}^{a}\right) \times \\
\left(u_{1}^{a} w_{2}^{b}-u_{2}^{b} w_{1}^{a}\right)\end{array}$ \\
\hline
\end{tabular}


In each of these tables the 3 trees appear in a chain of equivalences as in the Chain Equivalences Lemma 5.3. Therefore they are mutually equivalent. As before to prove that their sum is cancelable it is enough to prove that the sums of the coefficients in each table is even. Again a direct computation using the fact that $\omega(u \wedge w)=1$ yields the result.

The remaining trees are:

\begin{tabular}{|c|c|c|}
\hline \multirow{2}{*}{ Tree } & $4 b_{1}^{a_{1}} b_{1}$ & $\left.4\right|_{2} ^{a_{1}}$ \\
\cline { 2 - 3 } Coefficient & $\begin{array}{c}\left(b_{2}^{a} w_{2}^{b}-u_{2}^{b} w_{1}^{a}\right) \times \\
\left(u_{2}^{a} w_{1}^{b}-u_{1}^{b} w_{2}^{a}\right)\end{array}$ & $\begin{array}{c}a_{2} \\
\left.u_{1}^{a} w_{2}^{a}-u_{2}^{a} w_{1}^{a}\right) \times \\
\left(u_{2}^{b} w_{1}^{b}-u_{1}^{b} w_{2}^{b}\right)\end{array}$ \\
\hline
\end{tabular}

The IHX relation applied for instance to the first tree shows that the difference of the above two trees is cancelable. Therefore the sum of the two trees is equivalent to the first tree with coefficient the sum of the coefficients.

Summing the two cancelable brackets

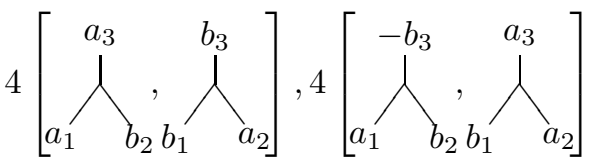

one finds that

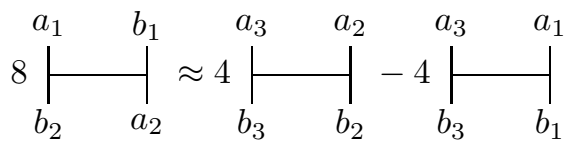

is cancelable by the Column Intersection Lemma 5.2 .

Therefore it is enough to show that the aforementioned sum of coefficients is even; again this is proved by direct computation.

5.3. Images of genus 2 twists are in $\operatorname{Can}\left(\mathcal{A}_{2}\left(N_{1}\right)\right)$. As one can see from Theorem 4.3 it remains to prove:

Theorem 5.3. Assume $g \geq 9$. For any elements $u_{1}, w_{1}, u_{2}, w_{2} \in N_{1}$ such that $\omega\left(u_{1} \wedge w_{1}\right)=1=\omega\left(u_{2} \wedge w_{2}\right)$, and $\omega\left(u_{1} \wedge w_{2}\right) \omega\left(u_{1} \wedge u_{2}\right)=0=\omega\left(w_{1} \wedge u_{2}\right)=$ $\omega\left(w_{1} \wedge w_{2}\right)$, the tree

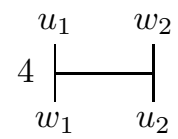

is cancelable.

Proof. As in the genus 1 case, using a suitable element in $\mathrm{GL}_{g}(\mathbf{Z})$ we may suppose that $u_{1}, u_{2}, w_{1}, w_{2} \in\left\langle a_{i}, b_{i} \mid 1 \leq i \leq 8\right\rangle$.

Write $u_{1}$ as $\widetilde{a}+\widetilde{b}$ with $\widetilde{a} \in L_{A}$ and $\widetilde{b} \in L_{B}$. Then

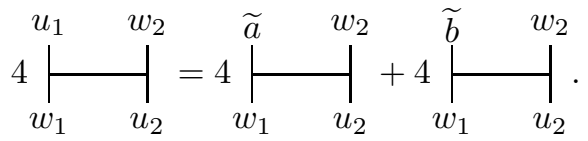


Computing the sum of the two cancelable brackets

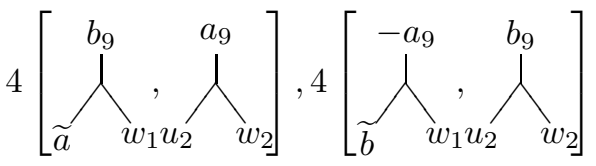

we get that

$$
\begin{aligned}
& \left.\left.4\right|_{w_{1}} ^{u_{1}}\right|_{u_{2}} ^{w_{2}} \approx-\left.\left.4 \omega\left(\widetilde{a} \wedge u_{2}\right)\right|_{b_{9}} ^{w_{1}}\right|_{a_{9}} ^{w_{2}}+\left.\left.4 \omega\left(\widetilde{a} \wedge w_{2}\right)\right|_{b_{9}} ^{w_{1}}\right|_{a_{9}} ^{u_{2}} \\
& +\left.\left.4 \omega\left(\widetilde{b} \wedge u_{2}\right)\right|_{a_{9}} ^{w_{1}}\right|_{b_{9}} ^{w_{2}}-\left.\left.4 \omega\left(\widetilde{b} \wedge w_{2}\right)\right|_{a_{9}} ^{w_{1}}\right|_{b_{9}} ^{u_{2}} .
\end{aligned}
$$

The equations $\omega\left(\widetilde{a} \wedge u_{2}\right)+\omega\left(\widetilde{b} \wedge u_{2}\right)=0=\omega\left(\widetilde{a} \wedge w_{2}\right)+\omega\left(\widetilde{b} \wedge w_{2}\right)$ imply that

$$
\begin{aligned}
& \left.\left.4\right|_{w_{1}} ^{u_{1}}\right|_{u_{2}} ^{w_{2}} \approx \omega\left(\widetilde{b} \wedge u_{2}\right)(\left.\left.4\right|_{a_{9}} ^{w_{1}}\right|_{b_{9}} ^{w_{2}}+\left.4 \underbrace{}_{b_{9}}\right|_{a_{9}} ^{w_{1}}) \\
& +\omega\left(\widetilde{a} \wedge w_{2}\right)\left(4{\underset{b}{b_{9}}}_{a_{9}}^{w_{1}}+4{\underset{a_{9}}{n_{b_{9}}}}_{n_{2}}^{u_{1}}\right) .
\end{aligned}
$$

We only prove that $\left.4\right|_{a_{9}} ^{w_{1}}+b_{9}+\underbrace{w_{2}}_{b_{9}}+\left.\right|_{a_{9}} ^{w_{1}}$ is cancelable; the proof for the remaining sum is the same.

Applying the IHX relation to this sum we get that

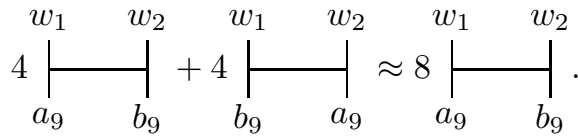

Expanding by multilinearity $\left.8\right|_{a_{9}} ^{w_{1}}$, we find that it is the sum of the following trees, where $1 \leq i \neq j \leq 8$, up to a multiplicative coefficient which is irrelevant to the proof:

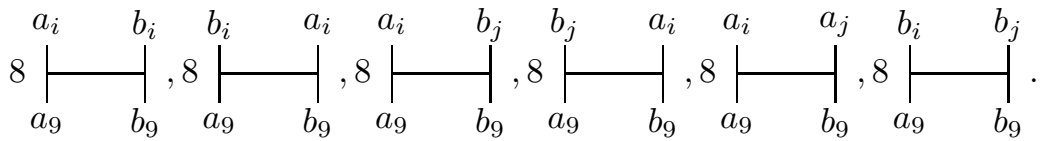

Applying the IHX relation to the first two trees we find that they are both the sum of cancelable trees. The last 4 trees are each equal to 2 times a tree that is involved in a chain of equivalences (cf. Lemma 5.3), hence they are all cancelable. 


\section{ACKNOWLEDGEMENTS}

The author would like to thank Professor Jean Barge for his support during the different stages of this work. This paper also benefited from various mathematical discussions on its subject and many others with Professors Jacques Thévenaz from the University of Lausanne, Pierre de la Harpe from the University of Geneva and Jérôme Scherer from the Universitat Autónoma de Barcelona.

\section{REFERENCES}

1. D. Bar-Natan, On the Vassiliev knot invariants, Topology 34 (1995), no. 2, 423-472. MR1318886 (97d:57004)

2. R. Craggs, A new proof of the Reidemeister-Singer theorem on stable equivalence of Heegaard splittings, Proc. Amer. Math. Soc. 57 (1976), no. 1, 143-147. MR0410749 (53:14495)

3. S. Garoufalidis and J. Levine, Tree-level invariants of three-manifolds, Massey products and the Johnson homomorphism, Graphs and patterns in mathematics and theoretical physics, Proc. Sympos. Pure Math., vol. 73, Amer. Math. Soc., Providence, RI, 2005, pp. 173-203. MR2131016 (2005m:57017)

4. H. B. Griffiths, Automorphisms of a 3-dimensional handlebody, Abh. Math. Sem. Univ. Hamburg 26 (1963/1964), 191-210. MR0159313 (28:2530)

5. N. Habegger and G. Masbaum, The Kontsevich integral and Milnor's invariants, Topology 39 (2000), no. 6, 1253-1289. MR1783857 (2002b:57011)

6. N. Habegger and W. Pitsch, Tree level Lie algebra structures of perturbative invariants, J. Knot Theory Ramifications 12 (2003), no. 3, 333-345. MR1983089 (2004e:57017)

7. Morris W. Hirsch, Differential topology, Graduate Texts in Mathematics, vol. 33, SpringerVerlag, New York, 1994, Corrected reprint of the 1976 original. MR1336822 (96c:57001)

8. D. Johnson, The structure of the Torelli group. II. A characterization of the group generated by twists on bounding curves, Topology 24 (1985), no. 2, 113-126. MR793178 (86i:57011)

9. J. Levine, Addendum and correction to: "Homology cylinders: an enlargement of the mapping class group" Algebr. Geom. Topol. 1 (2001), 243-270; Algebr. Geom. Topol. 2 (2002), 11971204 (electronic). MR1943338 (2003k:57022); MR1823501 (2002m:57020)

10. W. Magnus, A. Karrass, and D. Solitar, Combinatorial group theory, second ed., Dover Publications Inc., Mineola, NY, 2004, Presentations of groups in terms of generators and relations. MR2109550 (2005h:20052)

11. S. Morita, Casson's invariant for homology 3-spheres and characteristic classes of surface bundles. I, Topology 28 (1989), no. 3, 305-323. MR1014464 (90h:57020)

12. Abelian quotients of subgroups of the mapping class group of surfaces, Duke Math. J. 70 (1993), no. 3, 699-726. MR1224104 (94d:57003)

13. J. Nielsen, Untersuchungen zur Topologie der geschlossenen zweiseitigen Flächen i, Acta Math 50 (1927), 189-358.

14. __ Untersuchungen zur Topologie der geschlossenen zweiseitigen Flächen II, Acta Math 53 (1929), 1-76.

15. U Untersuchungen zur Topologie der geschlossenen zweiseitigen Flächen III, Acta Math 58 (1931), 87-167.

16. W. Pitsch, Une construction intrinsèque du cour de l'invariant de Casson, Ann. Inst. Fourier (Grenoble) 51 (2001), no. 6, 1741-1761. MR1871288 (2002k:57034)

17. Extensions verselles et automorphismes des groupes nilpotents libres, J. Algebra 249 (2002), no. 2. MR1901170 (2003h:20047)

18. K. Reidemeister, Zur dreidimensionalen Topologie, Abh. Math. Sem. Univ. Hamburg (1933), 189-194.

19. J. Singer, Three-dimensional manifolds and their Heegaard diagrams, Trans. Amer. Math. Soc. 35 (1933), no. 1, 88-111. MR1501673

20. S. Suzuki, On homeomorphisms of a 3-dimensional handlebody, Canad. J. Math. 29 (1977), no. 1, 111-124. MR0433433 (55:6409)

Departament de Matemàtiques, Universitat Autònoma de Barcelona, E-08193 BelLATERRA, SPAIN

E-mail address: pitsch@mat.uab.es 